\title{
Sensor fusion methods for high performance active vibration isolation systems
}

\author{
C. Collette ${ }^{\mathrm{a}, *}$, F. Matichard ${ }^{\mathrm{b}, \mathrm{c}}$ \\ a Université Libre de Bruxelles, BEAMS department, 50, F.D. Roosevelt av., 1050 Brussels, Belgium \\ ${ }^{\mathrm{b}}$ Massachusetts Institute of Technology, 185 Albany St., NW22-295 Cambridge, MA 02139, United States

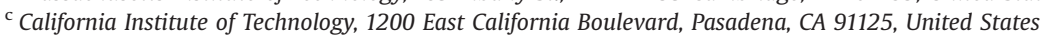

\section{A R T I C L E I N F O}

\section{Article history:}

Received 12 April 2014

Received in revised form

2 December 2014

Accepted 6 January 2015

Handling Editor: D.J. Wagg

Available online 30 January 2015

\begin{abstract}
A B S T R A C T
Sensor noise often limits the performance of active vibration isolation systems. Inertial sensors used in such systems can be selected through a wide variety of instrument noise and size characteristics. However, the most sensitive instruments are often the biggest and the heaviest. Consequently, high-performance active isolators sometimes embed many tens of kilograms in instrumentation. The weight and size of instrumentation can add unwanted constraint on the design. It tends to lower the structures natural frequencies and reduces the collocation between sensors and actuators. Both effects tend to reduce feedback control performance and stability. This paper discusses sensor fusion techniques that can be used in order to increase the control bandwidth (and/or the stability). For this, the low noise inertial instrument signal dominates the fusion at low frequency to provide vibration isolation. Other types of sensors (relative motion, smaller but noisier inertial, or force sensors) are used at higher frequencies to increase stability. Several sensor fusion configurations are studied. The paper shows the improvement that can be expected for several case studies including a rigid equipment, a flexible equipment, and a flexible equipment mounted on a flexible support structure.
\end{abstract}

(c) 2015 Elsevier Ltd. All rights reserved.

\section{Introduction}

In many applications, active systems are required to isolate sensitive equipment from input motion disturbance (e.g. ground floor, or base motion, environmental disturbances) [1,2]. High performance isolation required for sensitive applications is often reached by combining high loop gain feedback control and very low noise inertial sensors, like seismometers and geophones $[3,4]$. In order to have sufficient stability margins, it is common practice to collocate sensors and actuators, which ensures that the open loop transfer function has alternating poles and zeros along the imaginary axis $[5,6]$. As a consequence, each phase lag introduced by the poles is compensated by phase lead of the same amount introduced by the zeros, which guarantees stability. Such feedback systems are sometimes referred to as hyperstable [7]. However, in practice, several factors can cause phase loss, including analog-to-digital converters, dynamics of sensors and actuators [8], or resonances involving flexibility between the actuator and the sensor [9]. Under these practical constraints, the stability becomes conditional. Even with a careful design, the flexibility between sensors and actuators remains difficult

\footnotetext{
* Corresponding author.

E-mail address: ccollett@ulb.ac.be (C. Collette).
} 
(a)

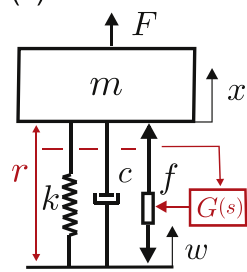

(b)

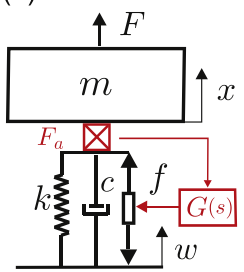

(c)

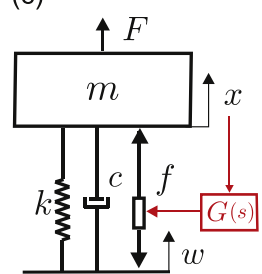

Fig. 1. Active isolators represented by single d.o.f. system: (a) relative motion control; (b) force control; (c) inertial control.

to avoid at high frequency (typically above $100 \mathrm{~Hz}$ ), especially when large and heavy sensors must be used to achieve very low noise isolation performance [10]. Such modes, known as in-the-loop modes [11], are the topic of this paper. In order to limit the effect of these modes, a conservative approach in the controller design is to limit the bandwidth to well below the lowest structural natural frequencies. However, when larger gains are required, the resonances must be adequately filtered out of the open loop transfer function. A first possibility is to damp them passively, e.g. with Dynamic Vibration Absorbers (DVA) $[12,13]$. However, DVA may be difficult to design for some applications, and their efficiency may be limited. Another possibility is to use the so-called plant inversion and notch filtering techniques [14,10]. The major drawback of these techniques is that they depend on the knowledge of the system, and thus, they are sensitive to plant variations.

Sensor fusion techniques have been used in active vibration isolators to combine the benefits of different types of sensors. They can combine relative sensor providing DC positioning capability at low frequency with inertial sensors providing isolation at higher frequency [15]. In other applications, they are used to combine inertial sensor at low frequency with force sensor at higher frequency to improve the robustness [16], or damp the internal modes [17,18].

In this paper, we study and compare different sensor fusion methods combining inertial sensors at low frequency with sensors adding stability at high frequency. The new signal resulting from the fusion of the inertial sensor and the highfrequency sensor will be called a super sensor. We will show that the stability margins of the controller can be significantly increased with no or little effect on the low-frequency active isolation, provided that the two following conditions are fulfilled: (i) the high-frequency sensor and the actuator are dual [19] and (ii) there exists a bandwidth where we can superimpose the open loop transfer functions obtained with the two sensors.

The paper is organized as follows: Section 2 reviews fundamental features and limitations of feedback control systems which use relative motion, force, or inertial sensors independently. Then the discussion is focused on inertial sensor's noise and size, the consequences for the design and the control bandwidth. Sections 3-5 investigate different types of high frequency sensor fusions and their impact on the stability of flexible equipment and flexible support structure. Section 6 draws the conclusions.

\section{Sensor type, noise and size}

This section discusses the effect of the sensor's nature (relative, inertial or force) on active vibration isolators' transmissibility and compliance. It also discusses the effect of the sensor noise on active isolation performance, and points out general trends regarding sensor noise versus sensor weight for typical inertial instruments.

\subsection{Relative motion control}

Feedback control based on relative sensors typically permits to servo-position a system or platform relative to a reference (e.g. floor or support base). It is illustrated in Fig. 1(a) for a single degree of freedom platform.

In the case of a vibration isolator, a negative feedback will tend to reduce the passive seismic isolation (only negative feedback will be considered in this paper). This is shown in Eqs. (1)- (4). Eq. (1) shows the motion $x$ of a platform of mass $m$, isolated from the ground motion $w$, thanks to springs (or otherwise passive suspension) components of overall stiffness $k$, and subjected to a disturbing force $F$. ${ }^{1}$ Viscous damping can be introduced to damp the resonance, but must be small enough to not compromise the high frequency vibration isolation. In this example, the feedback control force $f$ is controlled by a compensator $G(s)=g H(s)$ fed by a relative sensor signal $r=x-w$ as written in Eq. (2), where $H(s)$ is the controller filter, and $g$ is the controller gain. The compensator can be a PID or any higher-order controller providing high loop gain in the control bandwidth. Eq. (3) gives the closed loop transmissibility, and Eq. (4) gives the closed loop compliance. In the control bandwidth, high loop gain can be achieved, and the limit given in Eq. (5) shows that the controller "locks" the suspended stage with the ground, therefore suppressing the vibration isolation. Eq. (6) shows how the compliance is reduced by the controller:

$$
\left(m s^{2}+k\right) x=k w+f+F
$$

\footnotetext{
${ }^{1}$ Throughout the paper, the damping terms have been omitted to simplify the equations.
} 


$$
\begin{gathered}
\left(m s^{2}+k\right) x=k w-G(x-w)+F \\
\frac{x}{w}=\frac{k+G}{m s^{2}+k+G} \\
\frac{x}{\bar{F}}=\frac{1}{m s^{2}+k+G} \\
\lim _{G \rightarrow \infty} \frac{x}{W}=1 \\
\lim _{G \rightarrow \infty} \frac{x}{F}=\frac{1}{G}
\end{gathered}
$$

\subsection{Force control}

The actuator is fed with a measure of the total force, $F_{a}$, applied on the mass $m$ (Fig. 1(b)). The dynamics is still given by Eq. (1), but the control force is

$$
f=-G F_{a}=-G[f-k(x-w)]
$$

or

$$
f=\frac{G k}{1+G}(x-w)
$$

Substituting (7) in (1) gives

$$
m s^{2} x+\frac{k}{1+G}(x-w)=F
$$

the transmissibility

$$
\frac{x}{w}=\frac{k}{(1+G) m s^{2}+k}
$$

and the compliance

$$
\frac{x}{F}=\frac{1+G}{(1+G) m s^{2}+k}
$$

At low frequency, where the loop gain is expected to be high, the limits can be written to show the effect of the controller on the transmissibility and the compliance:

$$
\begin{aligned}
& \lim _{G \rightarrow \infty} \frac{x}{W}=\frac{k}{G m s^{2}} \\
& \lim _{G \rightarrow \infty} \frac{x}{F}=\frac{1}{m s^{2}}
\end{aligned}
$$

The isolator's overall stiffness has been effectively reduced by a factor $(1+G)$, which lowers the effective natural frequency, and therefore increases the isolation, but sacrifices system compliance in doing so.

\subsection{Inertial control}

Feedback control based on inertial sensing improves not only the vibration isolation but also the compliance. In Eq. (13), the compensator is fed with the absolute motion measurement $x$ produced by an inertial sensor, which can be a geophone, a seismometer, or an accelerometer (Fig. 1(c)).

For simplicity, the inertial sensor signal is assumed to be "stretched" (inverted) to provide a direct measure of the absolute motion $x$. The AC coupled nature of inertial sensors, and implication on noise and lower unity gain frequencies are discussed in the next sections. Eq. (14) shows the closed loop transmissibility, and Eq. (15) shows the closed loop compliance. The limit for high loop gain given in Eqs. (16) and (17) shows the suppression introduced by the inertial control. The inertial control increases the decoupling between the ground motion and the platform, but also reacts to force disturbances. In contrast to control with relative motion, both the transmissibility and the compliance have been reduced by the controller:

$$
\left(m s^{2}+k\right) x=k w-G x+F
$$




$$
\begin{gathered}
\frac{x}{w}=\frac{k}{m s^{2}+k+G} \\
\bar{F}=\frac{1}{m s^{2}+k+G} \\
\lim _{G \rightarrow \infty} \frac{x}{W}=\frac{k}{G} \\
\lim _{G \rightarrow \infty} \frac{x}{F}=\frac{1}{G}
\end{gathered}
$$

\subsection{In loop sensor noise}

One drawback of inertial sensors is that they are AC coupled and therefore sensor noise dominated at low frequencies. AC coupling characteristics imply a low unity gain frequency (or the use of a sensor fusion with a DC coupled sensor [15]). This is further discussed in Section 3.1. Regarding the sensor noise, Eqs. (18) and (19) show the influence of the sensor noise, $n_{x}$, on the system through the controller and the corresponding closed loop response (the disturbing force $F$ has been ignored for simplicity). The limit for high loop gain given in Eq. (20) shows that the platform motion tends to the opposite of the sensor noise:

$$
\begin{gathered}
\left(m s^{2}+k\right) x=k w-G\left(x+n_{x}\right) \\
x=\frac{k w-G n_{x}}{m s^{2}+k+G} \\
\lim _{G \rightarrow \infty} x=-n_{x}
\end{gathered}
$$

The minimal motion that can be obtained is therefore limited by the sensor noise. This statement can be generalized to systems with more complicated transfer functions as shown in the block diagram in Fig. 2, where $P_{s}$ is the seismic path, $P_{f}$ is the control force path, $S$ is the inertial sensor transfer function, $H$ is the control filter and $g$ is the control gain.

Eq. (21) gives the loop gain and Eq. (22) gives the closed loop response of the system. The limit for high loop gain given in (23) shows that the motion tends to the sensor noise independently of the frequency content of the transfer functions:

$$
\begin{gathered}
C=-g H P_{f} S \\
x=\frac{P_{S}}{1+C} w-\frac{C}{1+C} n_{x} \\
\lim _{C \rightarrow \infty} x=-n_{x}
\end{gathered}
$$

\subsection{Sensor size and noise}

Section 2.4 emphasized that sensor noise limits vibration isolation performance in applications where inertial sensors are used in the limit of high loop gain. It is therefore important to carefully select sensors for a given vibration isolation application. Inertial sensors are available in a broad variety of size and noise performance. Fig. 3 shows a few instruments: a Trillium T240 and Streckeisen STS2 seismometers, a Geotech GS13 and a Sercel L4C geophones, a Wilcoxon and two Endevco accelerometers. While all of them are first-choice instruments, they offer very different compromises between size, sensitive frequency range, cost, and noise performance. A seismometer's inertial masses are servo-controlled and offer the best noise performance at low frequencies. The geophones are very low noise instruments above their natural frequencies. The more compact accelerometers are sensitive at higher frequencies. Fig. 4 compares typical noise amplitude spectral densities for

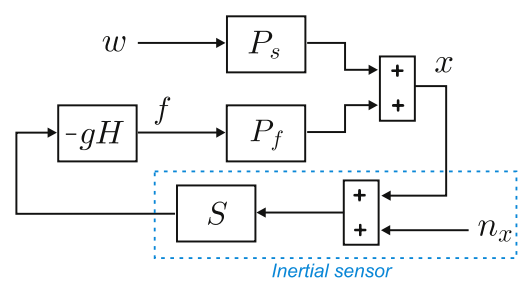

Fig. 2. Control using inertial sensor. 


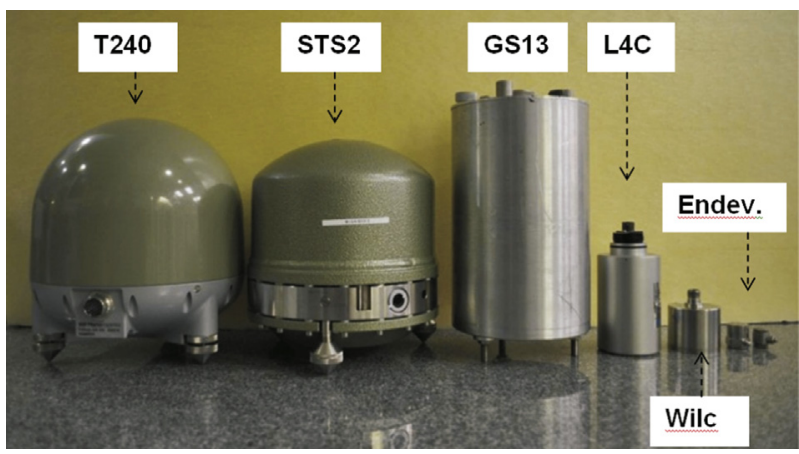

Fig. 3. Examples of inertial sensors used for active vibration isolation.

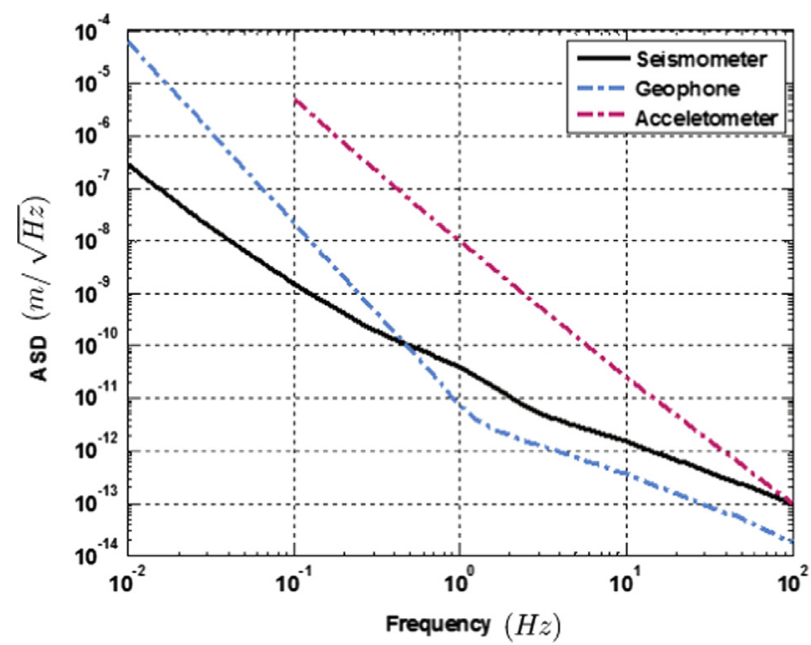

Fig. 4. Typical noise amplitude spectral density for very low noise Seismometers, Geophones and Accelerometers (Courtesy of Brian Lantz).

these low noise instruments. The complexity required to obtain low noise, low frequency performance implies that the more sensitive is the instrument, the larger and heavier it tends to be.

\subsection{Design constraints and control bandwidth}

The compromise between sensor noise and sensor size pointed out in the previous section has a direct impact on active vibration isolation system's design and their servo-control bandwidth. When low noise performance is needed at low frequencies, large instruments will be preferred.

However, they will put constraints on the design. First, it is harder to maintain collocation between sensors and actuators over large bandwidth when large sensors are used. This has a direct impact on the achievable control bandwidth, as discussed in the next sections. Then, the size and weight of sensors will put constraints on the platform's design. The rigidity of the structure and the modal content have a direct influence on the servo-controller bandwidth. The stiffer the structure, the easier it is to achieve high control bandwidth. Compared to smaller instruments, large and heavy sensor will tend to lower the structures natural frequencies, and therefore indirectly reduce the control bandwidth. Consequently, there is a subtle compromise to be obtained between sensor noise and the influence of the sensor size on the system's design and on the control bandwidth.

In the next sections, we study the combination of several types of sensors, to obtain both broadband low noise sensing and high bandwidth. The signal from the inertial sensor is used at low frequency. It is filtered by a low-pass filter $L_{p}$. The signal providing high-frequency stability is filtered by a high-pass filter $H_{p}$. The filters $L_{p}$ and $H_{p}$ are chosen to be complementary filters [15] to simplify the control loops design, i.e. $L_{p}+H_{p}=1$. The numerical values of the filters used in this paper are

$$
L_{p}=\frac{334867788.1472\left(s^{2}+213.3 s+2.274 e 004\right)}{(s+377)\left(s^{2}+380.3 s+9.389 e 004\right)\left(s^{2}+575.6 s+2.151 e 005\right)}
$$




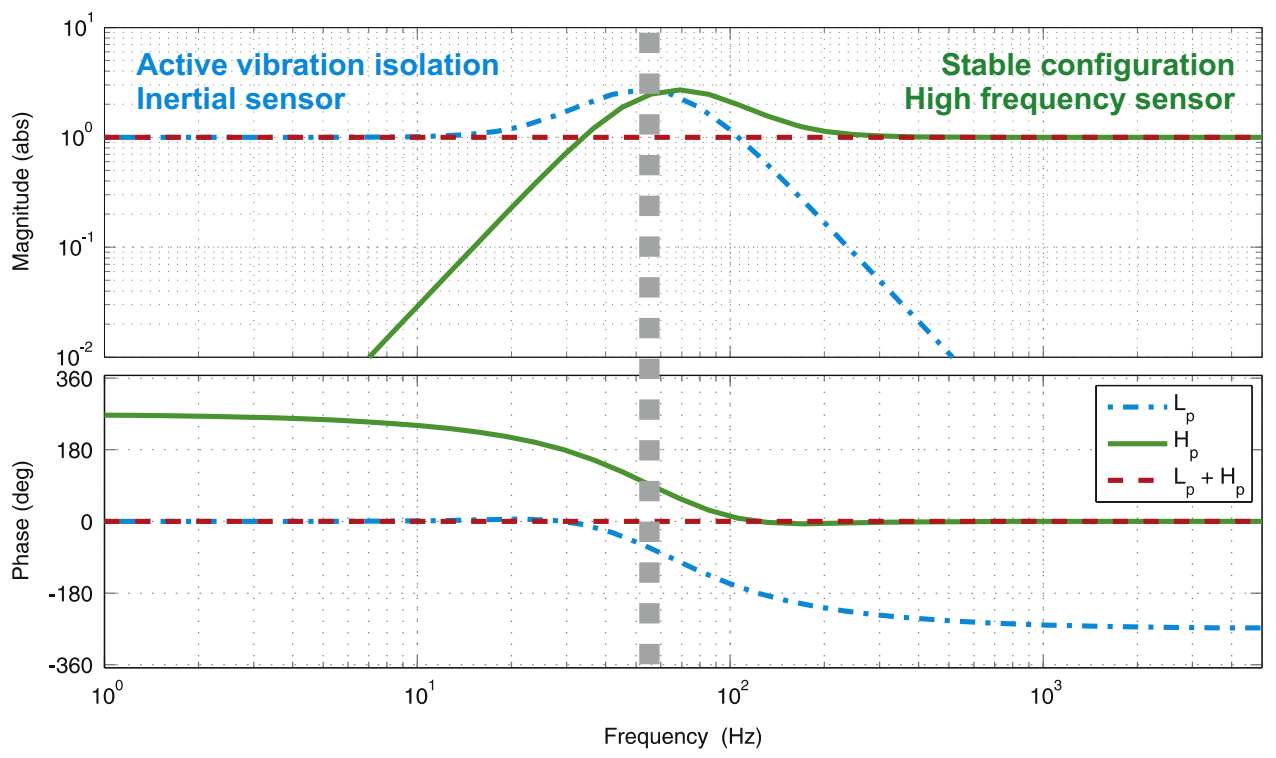

Fig. 5. Bode diagram of $L_{p}, H_{p}$ and $L_{p}+H_{p}$ which shows that the two filters are complementary.

$$
H_{p}=\frac{s^{3}\left(s^{2}+1333 s+8.883 e 005\right)}{(s+377)\left(s^{2}+380.3 s+9.389 e 004\right)\left(s^{2}+575.6 s+2.151 e 005\right)}
$$

Their frequency response is shown in Fig. 5. These filters will be used throughout the paper. The crossing frequency of the complementary filters is set slightly above the unity gain frequency of the control loop gain for the controllers used in the next sections. The order of $L_{p}$ and $H_{p}$ is a design parameter, which defines how the transition between the sensors occurs. The choice of the best order results from a tradeoff. As a general rule, a high order induces high amplitude of the complementary filters at the blend frequency. As will be shown, this may affect the closed loop performance around the blend frequency. On the other hand, the filter order has to be high enough for the sensor fusion to be fully dominated by the sensor of interest on either side of the blend frequency. In this study, third-order complementary filters have been chosen to ensure the inertial sensor signal dominates at low frequency, and the newly introduced sensor dominates at high frequency where we expect to improve performance. It results in an amplification of 2.8 around the blend frequency (Fig. 5). The effect of the amplification on the closed loop performance will be discussed later in the paper (e.g. bump in the compliance in Fig. 11b, or in the transmissibility in Fig. 14a).

Three types of sensors are studied to improve the high frequency stability:

- a smaller inertial sensor (e.g. a piezoelectric accelerometer), noisier at low frequency but easier to collocate with the actuator;

- a relative motion sensor dual of the actuator (hyperstable);

- a force sensor (also dual with the actuator/hyperstable).

In order to get a good physical insight, these three possibilities of high-frequency fusion will be studied on models with an increasing level of complexity.

\section{Inertial control and sensor fusion configurations}

While this paper addresses the problem of feedback control stability inherent to flexible structure models, this section uses a one degree of freedom model to introduce control and fusion techniques that will be used on flexible structures in the next sections. In this section, standard inertial-sensor-only control is discussed first for reference, and then the inertial sensor is fused with each sensor type to demonstrate the impact on transmissibility and compliance.

\subsection{Controller based on inertial sensing}

Fig. 1(c) shows the simplest model of an infinitely rigid and suspended structure, represented by a single degree of freedom (d.o.f.) isolator.

The absolute motion of the mass, $x$, is measured with an inertial sensor, considered as perfect (i.e. without internal dynamics and its velocity signal is integrated and calibrated into displacement units). It is important to point out that inertial sensors are inherently AC coupled which typically results in a lower unity gain frequency in the control loop. Fusion 
techniques can be used at low frequency to deal with this difficult problem [15] by combining the inertial sensor with a relative motion sensor. However, these low frequency issues and techniques are not discussed here to avoid confusion with the fusion techniques discussed later (to increase the high frequency stability margins). Therefore, the AC coupling nature of the inertial sensor is not represented in the following transfer functions. It is assumed that the inertial sensor transfer function has been perfectly stretched (inverted/integrated) down to the lowest frequency studied (10 $\mathrm{mHz}$ ). In practice, this would result in inertial sensor noise injection. Alternatively, low frequency sensor fusion with relative sensor can be used to compromise between sensor noise and isolation performance. In practical applications, the transmissibility would be somewhere between a few $\mathrm{dB}$ and the low level in the figures of this paper [11,21]. In this study, we focus on the high frequency noise and stability issues (near the upper unity gain frequency and above), and we purposely do not address the low frequency noise problem as it is already well covered in the literature [15]. The control force is driven by this perfectly calibrated inertial motion measurement through the compensator:

$$
f=-G(s)\left(x+n_{x}\right)=-g H(s)\left(x+n_{x}\right)
$$

where $n_{x}$ is the inertial sensor noise also calibrated in displacement units. The mechanical system's parameters are defined as follows to illustrate the discussion: $m=300 \mathrm{~kg}, 10 \mathrm{~Hz}$ natural frequency, and 1 percent of critical damping $(k=1.18 \mathrm{MN} / \mathrm{m}$ and $c=0.02 \sqrt{\mathrm{km}}=377 \mathrm{~N} \mathrm{~s} / \mathrm{m})^{2}$. A typical controller, shown in Fig. 6, is defined to support the discussion. It is composed of a lag to increase the loop gain at low frequency, a lead to have a sufficient phase margin at high frequency, and a gain value of $g=9 k$ has been chosen to set appropriate unity gain frequency just above $50 \mathrm{~Hz}$ :

$$
G(s)=g H(s)=9 k \frac{10(s+119.2)(s+10)}{(s+1192)(s+1)}
$$

Fig. 7 shows the corresponding open loop transfer function $(G x / f)$ and the root locus of this system. Substituting Eq. (24) in Eq. (1) gives

$$
x=\frac{k}{\left(m s^{2}+k+G\right)} w+\frac{1}{\left(m s^{2}+k+G\right)} F-\frac{G}{\left(m s^{2}+k+G\right)} n_{x}
$$

The isolator's transmissibility $(x / w)$ and compliance $(x / F)$ are shown in Fig. $8(\mathrm{a})$ and (b). They illustrate that the controller based on inertial sensing improves not only the compliance but also the transmissibility. However, structural flexibility discussed in Sections 4 and 5 will highlight stability issues that can arise with this control configuration.

\subsection{Inertial and force sensor}

A force sensor mounted between the suspended mass and the active suspension exhibits hyper-stability properties [6]. In this section we discuss the fusion of the inertial sensor signal with a force sensor using the same one d.o.f. model. The force sensor collocated with the actuator is mounted as shown in Fig. 9. The inertial sensor (large and heavy, but very sensitive) is used at low frequency where isolation performance is needed. The force sensor $F_{a}$ (noisier but forming a dual pair with the actuator) is used at higher frequency to improve the stability margins.

In this case, the control force is

$$
f=-G(s) S S_{F}=-g H(s) S s_{F}
$$

where $s s_{F}$ is called a super sensor, constructed from the fusion of the inertial sensor and the force sensor:

$$
s s_{F}=L_{p}\left(x+n_{x}\right)+H_{p}^{F}\left(F_{a}+n_{F}\right)
$$

where $n_{x}$ and $n_{F}$ are respectively the noise in the inertial sensor and the noise in the force sensor, calibrated in displacement and force units respectively, and

$$
H_{p}^{F}=\frac{H_{p}}{m s^{2}}
$$

is a filter combining the high pass complementary filter $H_{p}$ and a calibration factor to match the unit of the force sensor and inertial sensor (assumed to be in displacement units all along the text).

Fig. 10 shows the open loop transfer function between the actuator $f$ and the super sensor $s s_{F}$ normalized by stiffness $k$ for readability.

Replacing Eqs. (28) and (27) in (1), we get

$$
x=\frac{k}{\left(1+G H_{p}^{F}\right) m s^{2}+k+G L_{p}} w+\frac{1+G H_{p}^{F}}{\left(1+G H_{p}^{F}\right) m s^{2}+k+G L_{p}} F-\frac{G\left(L_{p} n_{x}+H_{p}^{F} n_{F}\right)}{\left(1+G H_{p}^{F}\right) m s^{2}+k+G L_{p}}
$$

\footnotetext{
${ }^{2}$ Experience shows that structures embedding heavy, low-frequency, low-noise instrumentation typically weighs several hundreds of kg to several tons. We picked the number $300 \mathrm{~kg}$ to illustrate the order of magnitude of the mass of such structures (a single, broadband, three-axis seismometer weighs roughly $15 \mathrm{~kg}$ ). We picked an intermediate fundamental resonance value of $10 \mathrm{~Hz}$, between soft suspensions isolators frequencies (around a $1 \mathrm{~Hz}$ or below) and stiff suspensions frequencies (around several tens of Hertz). Other values could have been arbitrarily picked and lead to the same conclusion in the simulation presented in the next section to illustrate the various sensor fusion methods.
} 


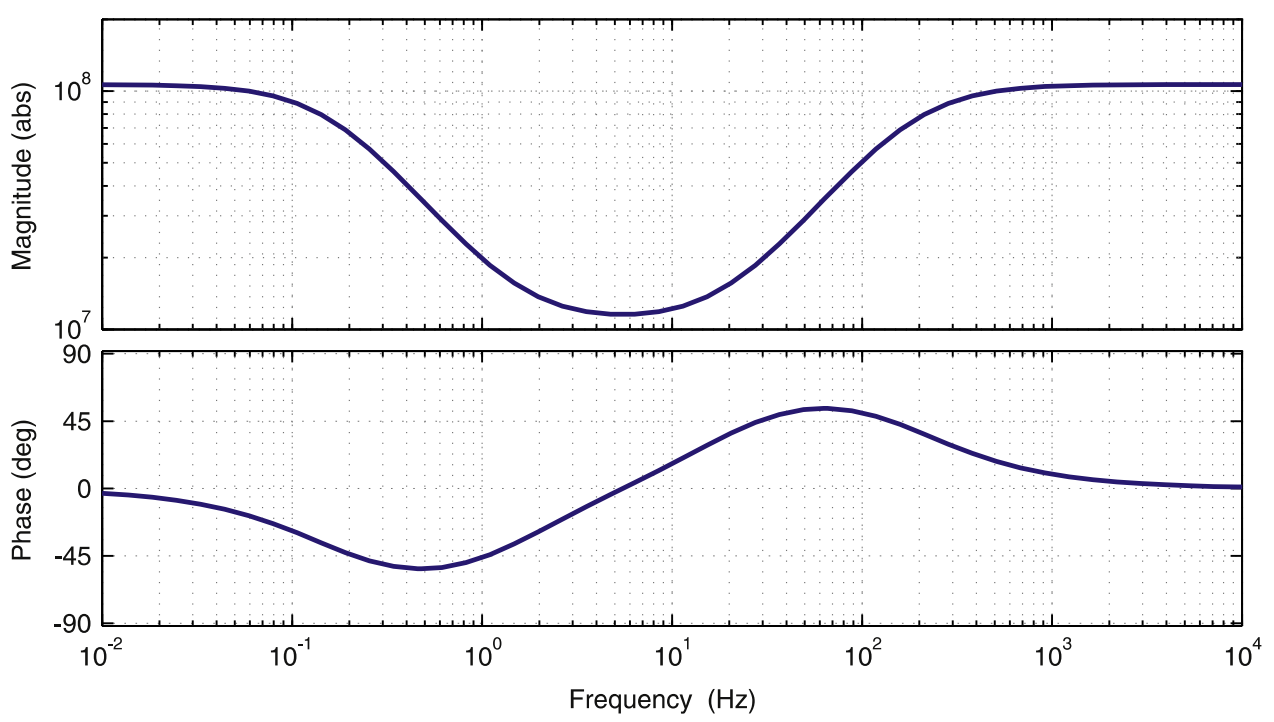

Fig. 6. Lead-lag controller $G(s)$ used for simulations.

(a)

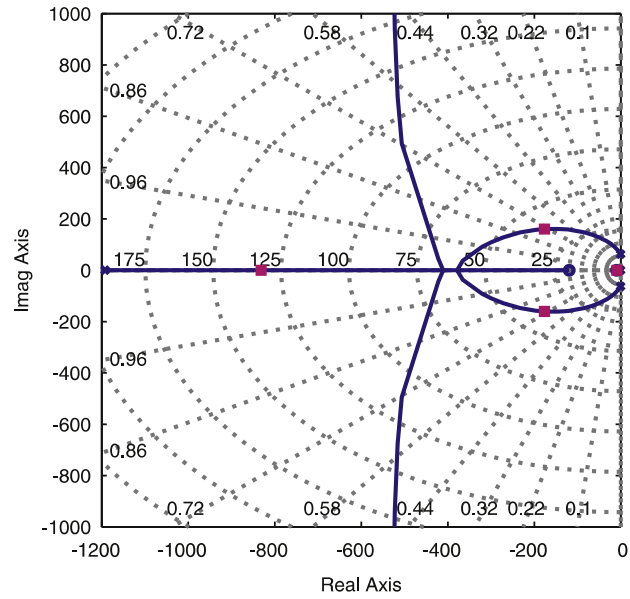

(b)

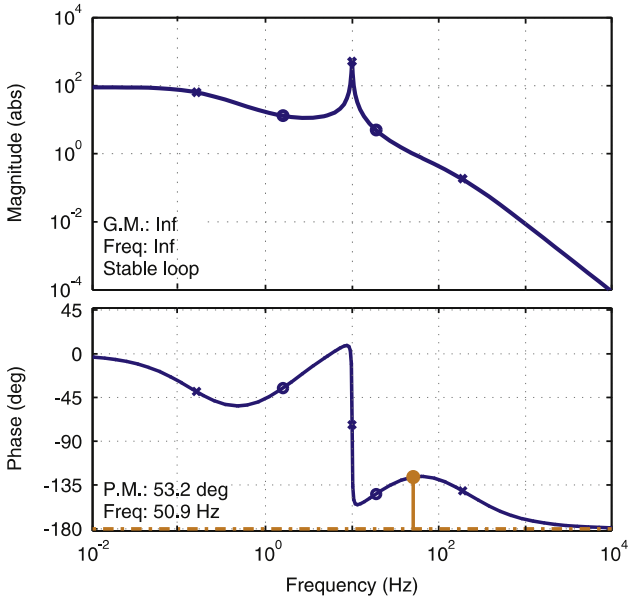

Fig. 7. Single d.o.f. isolator with inertial control: (a) root locus and (b) open loop transfer function.

which simplifies to

$$
x=\frac{k}{m s^{2}+k+G} w+\frac{1+G H_{p}^{F}}{m s^{2}+k+G} F-\frac{G\left(L_{p} n_{x}+H_{p}^{F} n_{F}\right)}{m s^{2}+k+G}
$$

The first term on the right-hand side of (31) shows that the transmissibility is unchanged (by comparison with the inertial control). The second term shows that the compliance is degraded by a factor $\left(1+G H_{p}^{F}\right)$. The third term shows the noise introduced by the force sensor. As an illustration, Fig. 11 shows the resulting closed loop transmissibility and compliance obtained using the controller defined in Section 3.1. The curves have been obtained with the same value of $g$ as before. The fusion filter can then be adjusted as a function of the application objectives to obtain a good compromise between sensor noise filtering and compliance degradation (i.e. more slope at low frequency in $H_{p}$ for better force sensor noise filtering would result in more amplification near the complementary filters crossover frequency).

\subsection{Inertial and relative sensor}

Control based on relative motion sensor tends to reduce the vibration isolation. However, this sensor form a dual pair with the actuator when both are collocated. Therefore, a sensor fusion can be implemented using the inertial sensor at low frequency (to provide isolation) and using the relative motion sensor at high frequency (to improve stability margins). The relative motion sensor is collocated with the actuator, as illustrated in Fig. 12. 
(a)

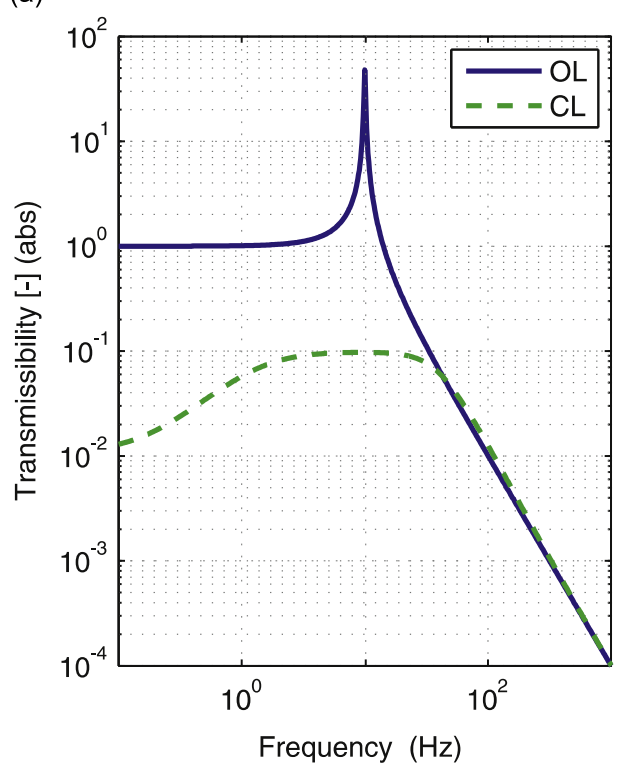

(b)

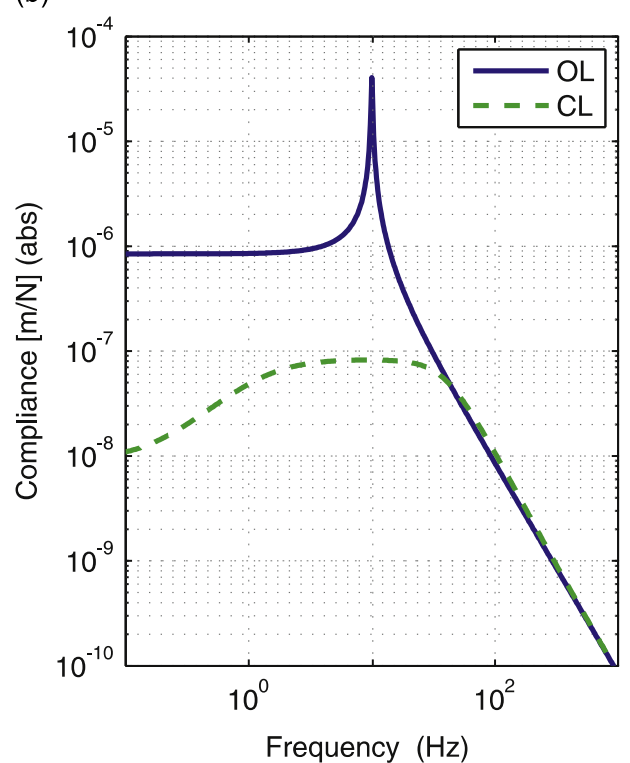

Fig. 8. (a) Transmissibility and (b) compliance of a single d.o.f. isolator in Open Loop (OL) and Closed Loop (CL) configuration.

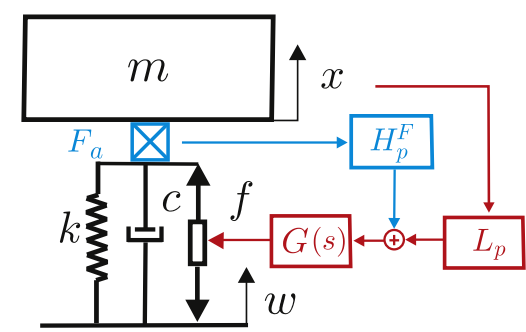

Fig. 9. Single d.o.f. isolator. Inertial and force control fusion.

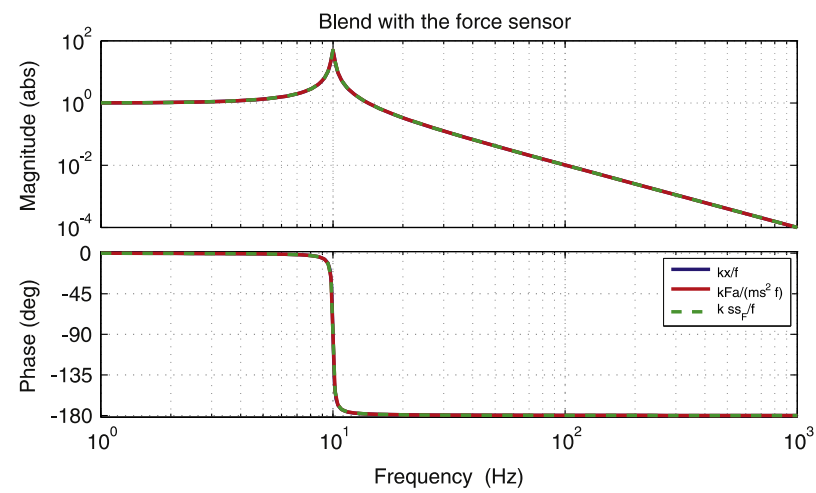

Fig. 10. Single d.o.f. isolator. Inertial sensor blended with a force sensor. Open loop transfer function $k x / f, k F_{a} /\left(m s^{2} f\right)$ and $k s s_{F} / f$.

In this case, Eq. (24) becomes

$$
f=-G s s_{r}=-g H(s) S s_{r}
$$

where

$$
s s_{r}=L_{p}\left(x+n_{x}\right)+H_{p}^{r}\left(r+n_{r}\right)
$$

where $r$ is the relative motion sensor and $n_{r}$ is its intrinsic noise, calibrated in displacement units. 
(a)

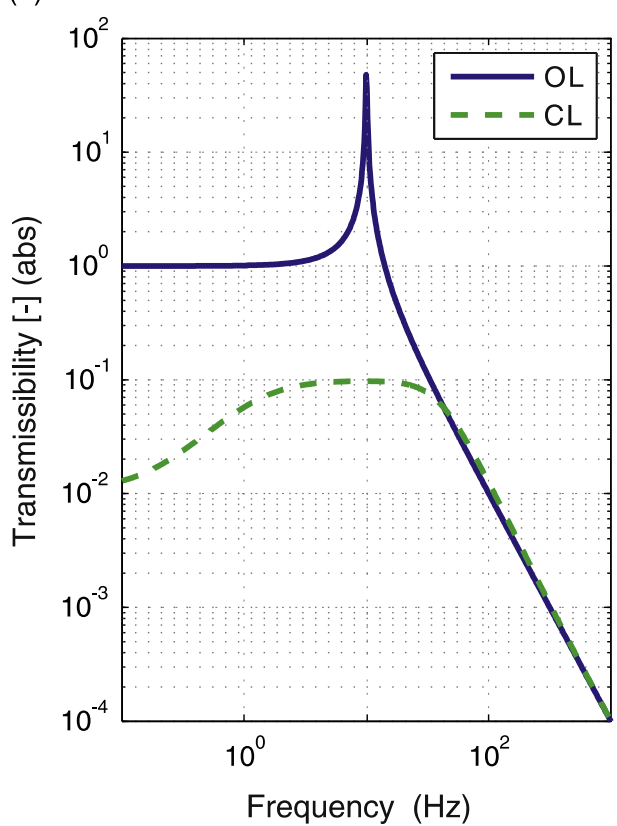

(b)

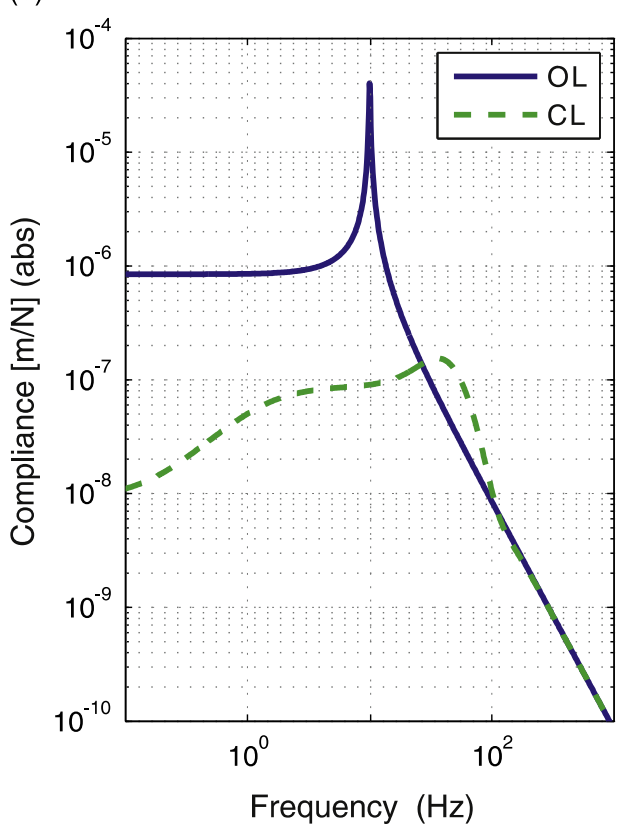

Fig. 11. Inertial sensor blended with a force sensor. (a) Transmissibility and (b) compliance of a single d.o.f. isolator in Open Loop (OL) and Closed Loop (CL) configuration.

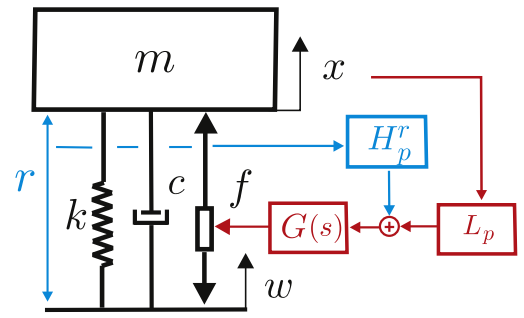

Fig. 12. Single d.o.f. isolator. Blend of an inertial sensor and a relative motion sensor.

The relative motion sensor can be either:

- A relative displacement sensor $r=x-w$. In this case,

$$
H_{p}^{r}=H_{p}
$$

- A relative velocity sensor $r=\dot{x}-\dot{w}$. In this case,

$$
H_{p}^{r}=\frac{H_{p}}{S}
$$

- A relative acceleration sensor $r=\ddot{x}-\ddot{w}$. In this case,

$$
H_{p}^{r}=\frac{H_{p}}{s^{2}}
$$

Fig. 13 shows the open loop transfer function between the actuator $f$ and the super sensor $s s_{r}$.

Replacing Eqs. (33) and (32) in (1), we get

$$
x=\frac{k+G H_{p}^{r}}{m s^{2}+k+G} w+\frac{1}{m s^{2}+k+G} F-\frac{G\left(L_{p} n_{x}+H_{p}^{r} n_{r}\right)}{m s^{2}+k+G}
$$




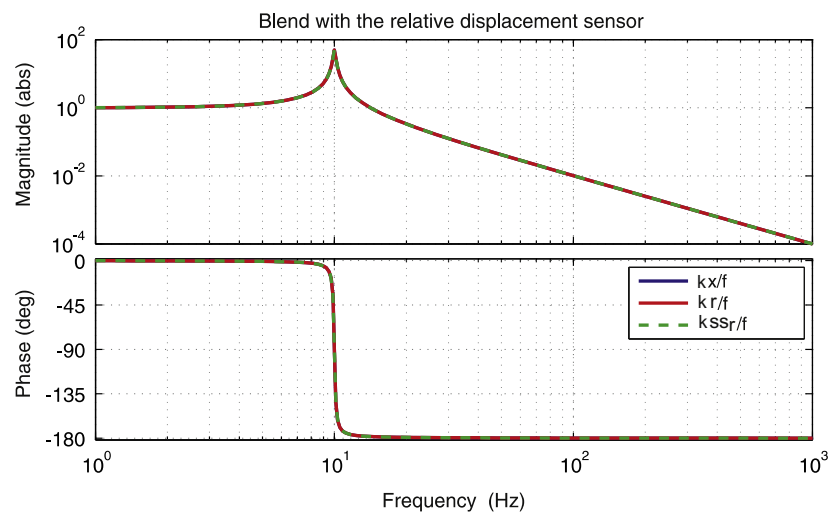

Fig. 13. Single d.o.f. isolator. Inertial sensor blended with a relative motion sensor. Open loop transfer function $k x / f, k r / f$ and $k s s_{r} / f$.

in which the fractions on the right-hand side are respectively the transmissibility, the compliance and the noise sensitivity. Compared to Eq. (26), one can be noticed that there is a significant degradation of the isolation at high frequency, because the relative sensor couples both sides of the actuator, while the compliance remains unchanged. The third term shows the noise introduced by the relative sensor.

Fig. 14 shows the resulting closed loop transmissibility and compliance. The curves have been obtained with the same value of $g$ as before.

Fusion with a relative motion sensor has no negative effect on the compliance, unlike fusion with a force sensor (Fig. 11(b)). However, compared to Fig. 11(a), the transmissibility shown in Fig. 14(a) has been degraded, due to the coupling introduced by the relative motion sensor. The blend filters can be tuned to change the compromise between isolation in the bandwidth and amplification outside. Nevertheless, this example illustrates the overall tendency. This approach can be of interest for systems using stiff suspensions and therefore providing little passive isolation, although the flexibility of the support structure must be carefully taken into account for the design of the blend filters (this is further discussed in Section 5).

In the next section, we will study the effect of the structure deformation on these sensor fusion methods. For that, a storage element (modeled by a spring) is introduced between the actuator and the sensor to study the stability of the feedback loop.

\section{Flexible equipment}

With the introduction of structural flexibility between the inertial sensor and actuator, the open loop gain transfer function can lose its hyper-stable properties with the same controller. In this section, we follow the same examples as in Section 3, but now including an additional degree of freedom to represent the impact of structure flexibility to demonstrate the impact of sensor fusion.

\subsection{Inertial sensor control}

A two d.o.f. system shown in Fig. 15 is introduced to represent the effect of structure's flexibility. Compared to Fig. 1(c), the mass of the isolator has been divided into two smaller masses, connected by a spring and a dashpot. This two mass system represents the flexibility of the structure between the location of the inertial sensor and the point on which the actuator applies a force on the structure.

In order to keep the results comparable with the case of the single d.o.f. system, the total mass is kept the same. It has been equally distributed between the two bodies. Another distribution could have been chosen, but would not impact the final results. The stiffness $k$ and damping ratio of the isolator suspension are unchanged. The model uses a stiffness $k_{1}=300 k=355 \mathrm{MN} / \mathrm{m}, c_{1}=0.02 \sqrt{k_{1} m / 2}=4617 \mathrm{~N} \mathrm{~s} / \mathrm{m}$ to be representative of a typical first deformation mode of structure. The dynamic equations of the system read

$$
\begin{gathered}
\frac{m}{2} s^{2} x+\left(k_{1}+s c_{1}\right)\left(x-x_{1}\right)=0 \\
\frac{m}{2} s^{2} x_{1}+\left(k_{1}+s c_{1}\right)\left(x_{1}-x\right)+(k+s c)\left(x_{1}-w\right)=f
\end{gathered}
$$

The control force is still given by Eq. (24). Fig. 16(a) and (b) shows the root locus and the open loop transfer function between the actuator and the sensor, using the same controller shown in Fig. 6. 
(a)

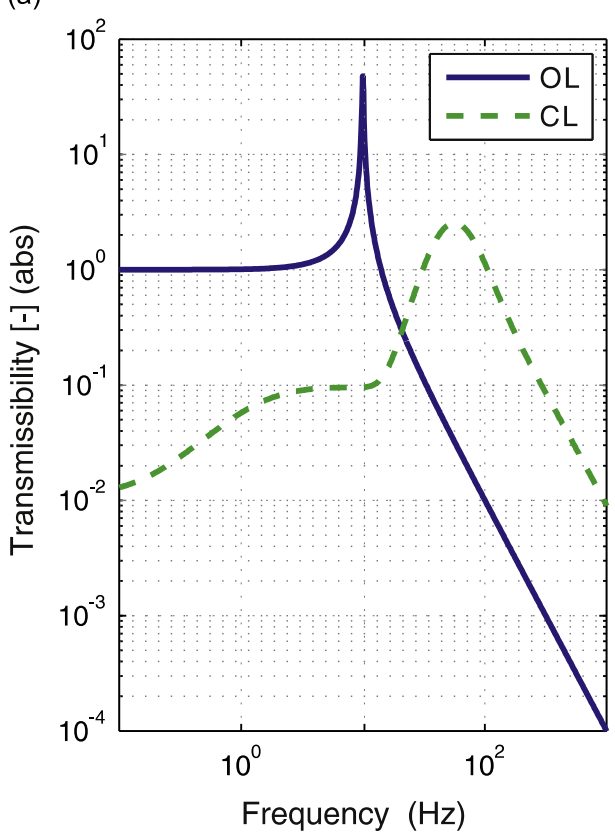

(b)

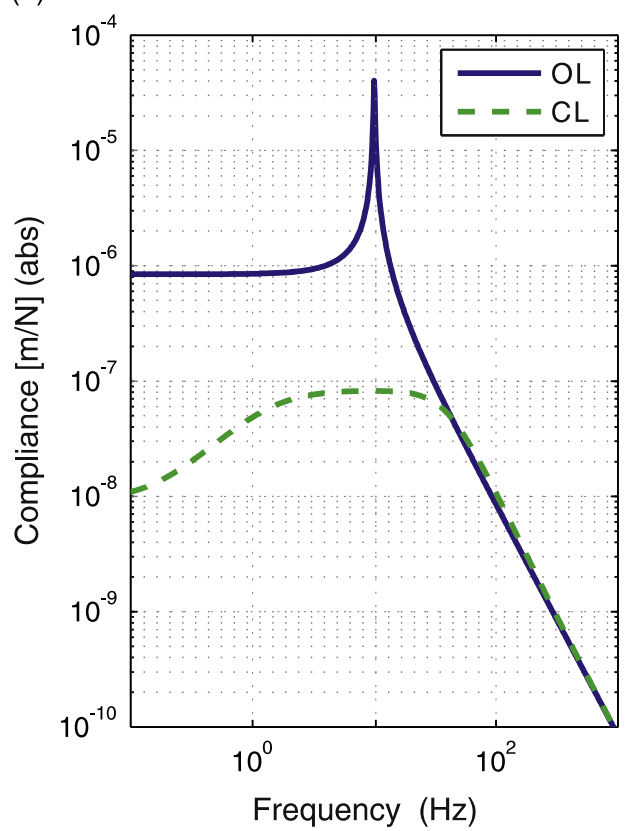

Fig. 14. Inertial sensor blended with a relative motion sensor. (a) Transmissibility and (b) compliance of a single d.o.f. isolator in Open Loop (OL) and Closed Loop (CL) configuration.

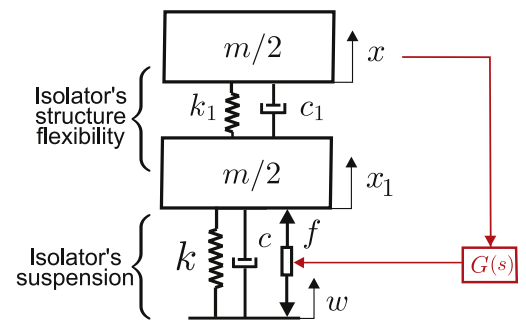

Fig. 15. Active isolator model including first structural mode.

(a)

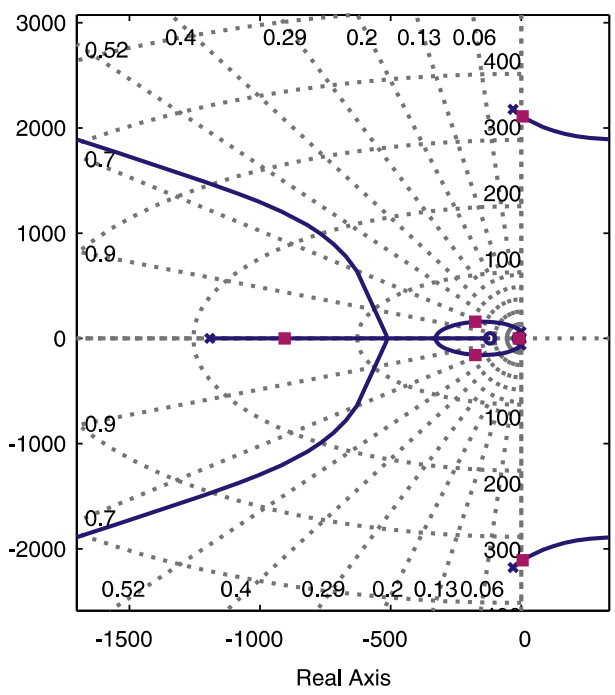

(b)

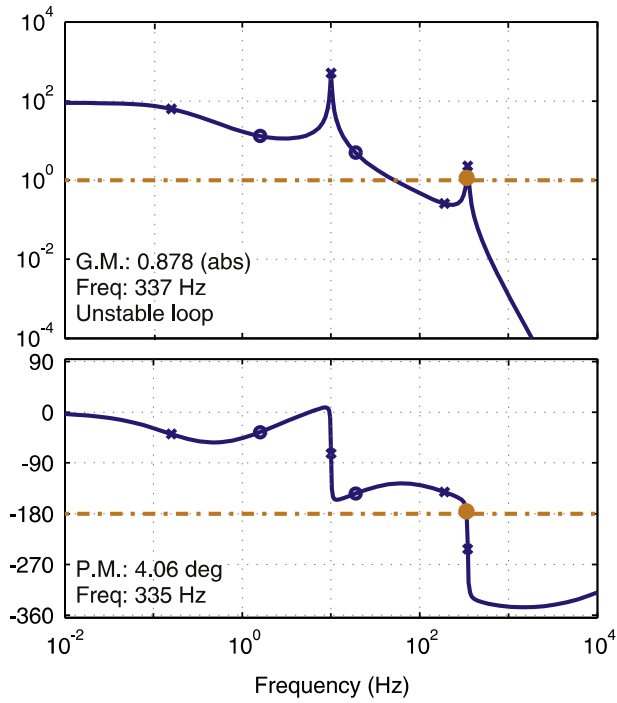

Fig. 16. Two d.o.f. isolator shown in Fig. 15. (a) Root locus and (b) open loop transfer function with the controller $G$ shown in Fig. 6 on the mass inertial displacement $x$. 
The system is now unstable, because there is no zero to restore the phase between two resonances. It is a direct consequence of the non-collocated configuration between the actuator and the sensor. The high frequency mode is known as a in-the-loop mode [11]. The system can still be stabilized by tuning the controller, via notching or plant inversion as mentioned in the Introduction. However, it is more difficult to obtain a sufficient phase margin, and a good robustness to model parameter variations. Further, this is an illustrative model with only one mode of compliance. Real structures may have many such modes making the control design implementation very complicated.

In the next three sections, we will blend the inertial sensor with another inertial sensor mounted closer to the actuator, with a force sensor and with a relative motion sensor. For each case, we will investigate the effect of the blend on the stability of the control loop.

\subsection{Inertial and small accelerometer}

Mounting a smaller inertial sensor near the actuator and fusing its signals with the distant low-frequency seismometer is one way to regain stability. This is illustrated in Fig. 17. In this example, the low-frequency inertial sensor (large and heavy) cannot be exactly collocated with the actuator. It senses the motion $x$. A smaller inertial sensor (an accelerometer in this example) is used to sense the absolute motion at the actuation point.

In this case, Eq. (24) becomes

$$
f=-g H(s) s s_{a}
$$

where the super sensor $s s_{a}$ is constructed from the blending of the inertial sensor $x$ and the accelerometer $a=\ddot{x}_{1}$ :

$$
s s_{a}=L_{p} x+H_{p}^{a} a
$$

and

$$
H_{p}^{a}=\frac{H_{p}}{s^{2}}
$$

where $L_{p}$ and $H_{p}$ are complementary filters shown in Fig. 5.

The normalized open loop transfer function $k x / f, k a /\left(s^{2} f\right)$ and $k s s_{a} / f$ are shown in Fig. 18. The main difference between $k x / f$ and $k s s_{a} / f$ is that a pair of zeros appeared in $k s s_{a} / f$ at high frequency (its value corresponds to the resonance of the

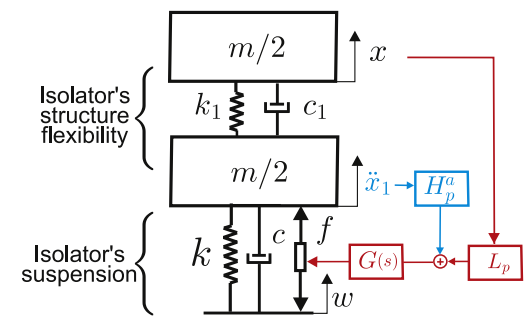

Fig. 17. Two d.o.f. isolator. Inertial sensor blended with an accelerometer.

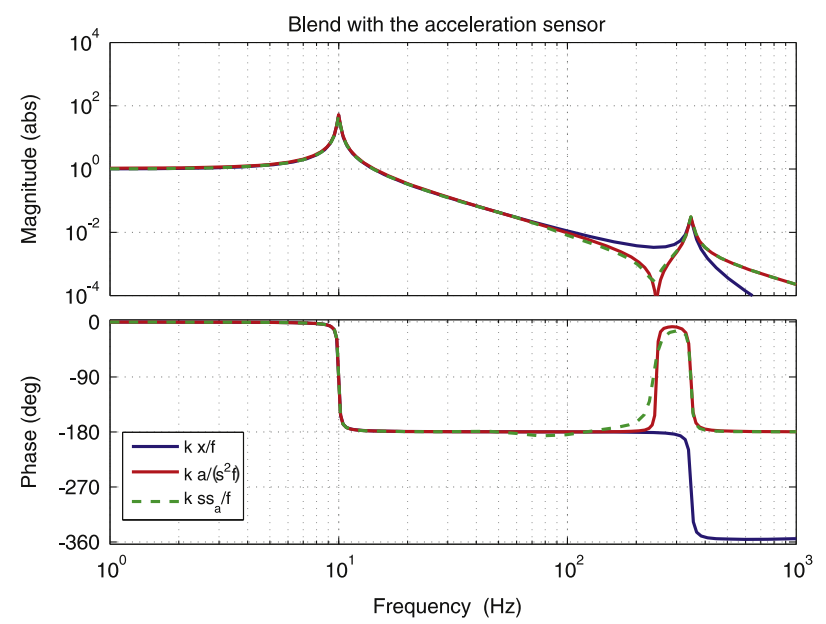

Fig. 18. Two d.o.f. isolator. Inertial sensor blended with a small accelerometer located close to the actuator. Open loop transfer function $k x / f$, $k a /\left(s^{2} f\right)$ and $k s s_{a} / f$. 
(a)

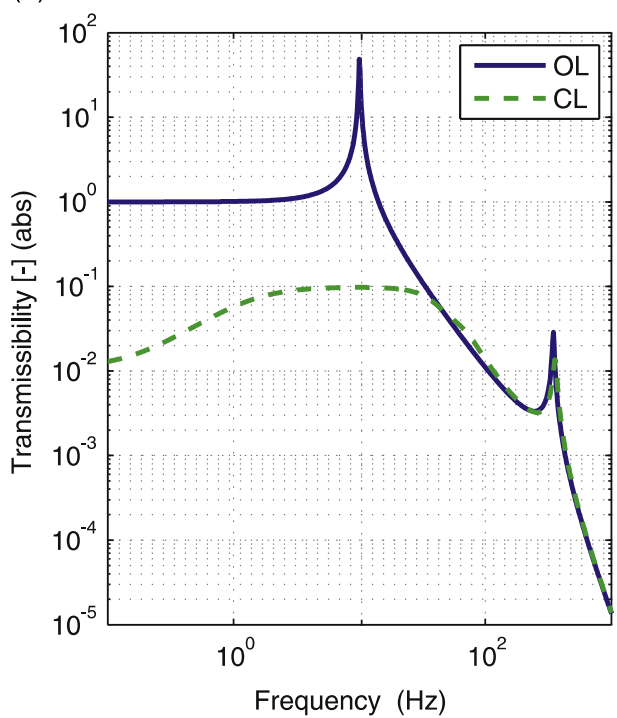

(b)

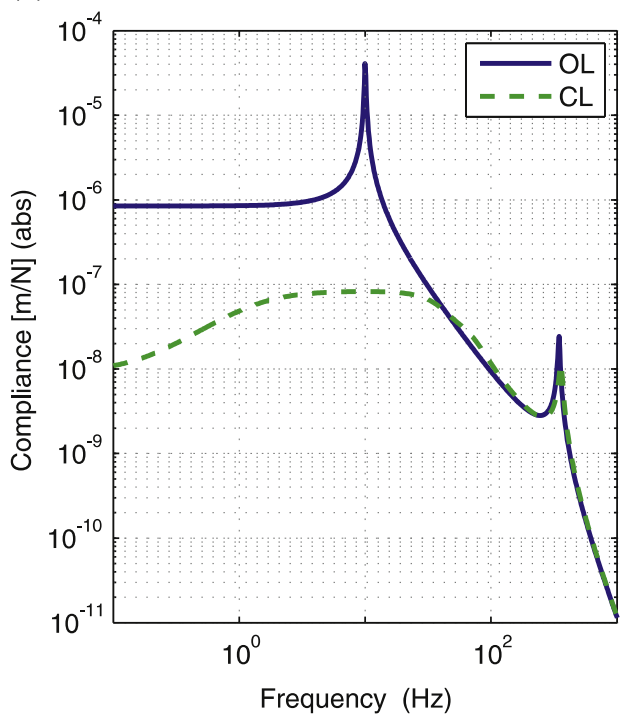

Fig. 19. Inertial sensor blended with a small accelerometer located close to the actuator. (a) Transmissibility and (b) compliance of a 2 d.o.f. isolator in Open Loop (OL) and Closed Loop (CL) configuration.

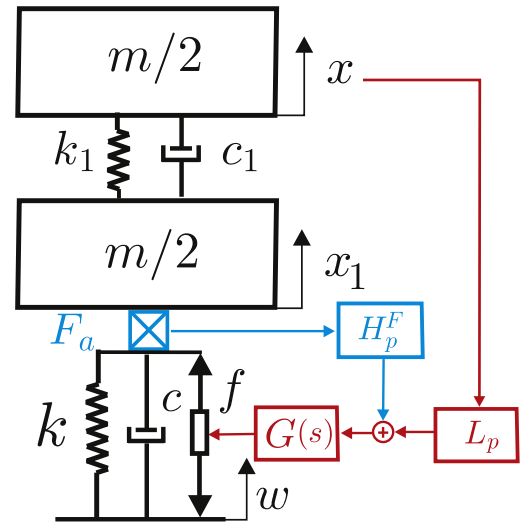

Fig. 20. Two d.o.f. isolator representing a flexible structure. Inertial sensor blended with a force sensor.

subsystem where $x_{1}$ is restrained). As a result, the phase remains bounded between $0^{\circ}$ and $-180^{\circ}$, which means that the use of an inertial sensor near the actuator permits to regain the phase stability. The transmissibility and compliance are shown in Fig. 19 in open loop and closed loop configuration.

In this example, fusion works very well because the frequency of the zeros is far enough from the blend frequency, i.e. the super sensor $s s_{a}$ is completely dominated by the accelerometer signal at high frequency. However, this is not a truly dual actuator/sensor configuration. Moreover, the smaller inertial sensors will be noisier than the large inertial sensor used to sense $x$. It is therefore important to verify that the noise introduced by the small inertial sensor does not compromise the vibration isolator performance (noise or error budgeting).

\subsection{Inertial and force sensor}

The fusion of the inertial and force sensor presented in Section 3.2 is now applied to the flexible structure to illustrate the benefits on loop shaping and stability. The inertial sensor is combined with a force sensor collocated with the actuator, as shown in Fig. 20.

The expression of the super sensor $s s_{F}$ is still given by Eq. (28) and the control force is also still given by Eq. (27). ${ }^{3}$

\footnotetext{
${ }^{3}$ In $[20,18]$, a similar controller has been proposed to damp the equipment modes, while here we are only interested in the controller stability at high frequency.
} 


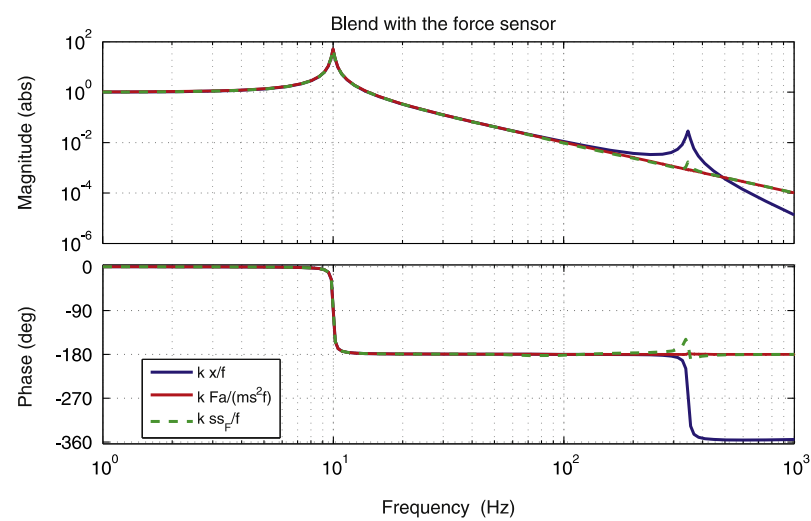

Fig. 21. Two d.o.f. isolator shown in Fig. 20. Inertial sensor blended with a force sensor. Open loop transfer functions $k x / f, k F_{a} /\left(m s^{2} f\right)$ and $k s s_{F} / f$.

(a)

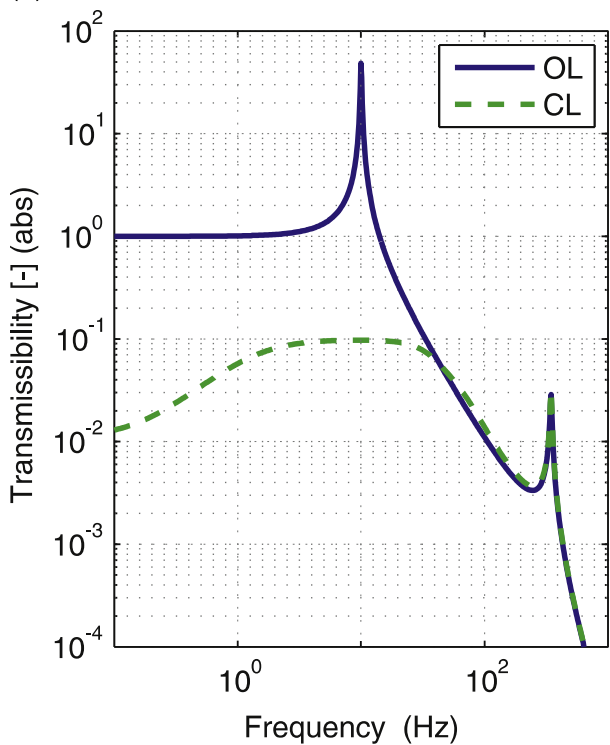

(b)

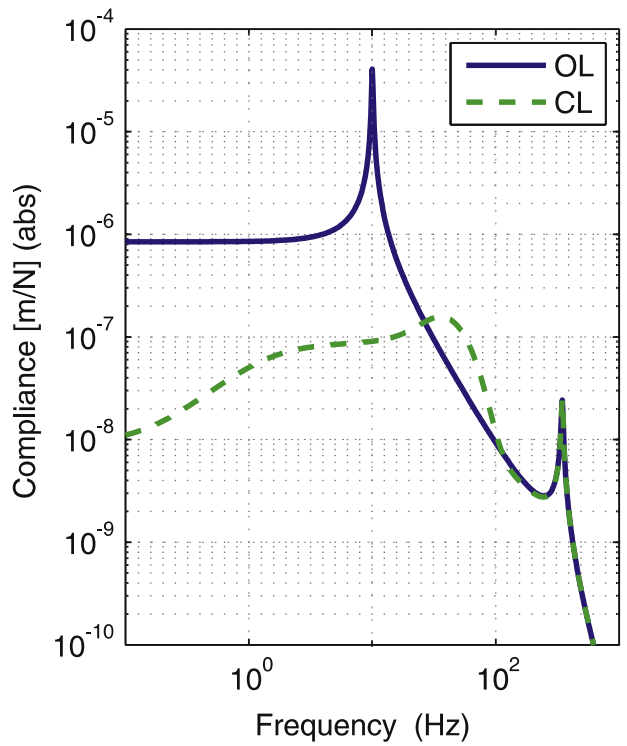

Fig. 22. Inertial sensor blended with a force sensor. (a) Transmissibility and (b) compliance of a 2 d.o.f. isolator in Open Loop (OL) and Closed Loop (CL) configuration.

Fig. 21 shows the open loop transfer functions $k x / f, k F_{a} /\left(m s^{2} f\right)$ and $k s s_{F} / f$. At low frequency, $k s s_{F} / f$ is dominated by the absolute motion sensor; at high frequency, it is dominated by the force sensor. The figure shows that the phase lag stops at $-180^{\circ}$, because a pair of zeros appeared just before the second pair of poles and cancels the poles. The frequency of the zeros corresponds to the resonance of a subsystem made of the two masses connected by $k_{1}$ and $c_{1}$ only (not connected to $k$ and $c$ ). In this example, the zero nearly cancels the third pole because $k$ is significantly lower than $k_{1}$.

Fig. 22 shows the resulting open loop and closed loop transmissibility and compliance curves.

This example illustrates that this fusion technique simplifies the loop shaping of the controller because the open loop is less sensitive to the deformation mode. It also highlights that the transmissibility is unchanged, at the cost of very little deterioration of the compliance. The impact of the fusion on the compliance is once again directly related to the order and shape of the complementary filters. Lower-order filters would further reduce the compliance at the blend frequency at the cost of additional noise from the force sensor compromising the isolation performance at low frequencies.

\subsection{Inertial and relative sensor}

The fusion of the inertial and relative sensor presented in Section 3.3 is now applied to the flexible structure to illustrate the benefits on stability. Consider the flexible structure shown in Fig. 23, where a relative sensor has been mounted in order to measure the elongation of the actuator.

The control law is still given by Eq. (32), and the super sensor by Eqs. (33)- (36). Fig. 24 shows the open loop transfer function $k x / f, k r / f$ and $k s s_{r} / f$. 


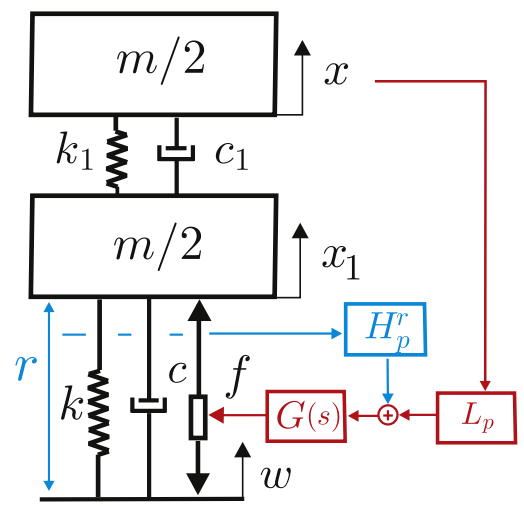

Fig. 23. Two d.o.f. isolator representing a flexible structure. Blend of an inertial sensor and a relative motion sensor.

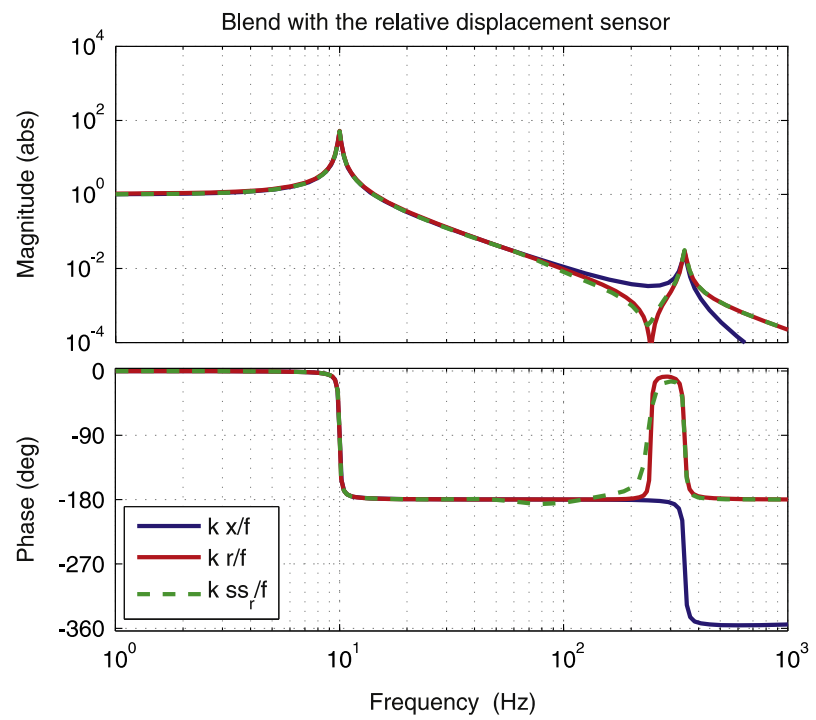

Fig. 24. Two d.o.f. isolator representing a flexible structure. Inertial sensor blended with a relative displacement sensor. Open loop transfer functions $k x / f$, $k r / f$ and $k s s_{r} / f$.

Fig. 25 shows the transmissibility and compliance, in open loop and closed loop configuration. The fusion permits to regain stability. However, compared to Fig. 22(a), the relative sensor has introduced a coupling between both sides of the actuator, which induces a significant degradation of the isolation at high frequency, already observed in the case of an ideal structure. On the other hand, the compliance (Fig. 25(b)) remains unchanged at high frequency.

\section{Flexible support structure and equipment}

\subsection{Inertial sensor control}

In this section, the effect of the flexibility of the support structure located under the active isolation stage is investigated. The support structure is represented in Fig. 26 by a mass chosen as $m / 2$ of motion $x_{0}$ with an internal stiffness and damping modeled with a spring constant chosen as $k_{0}=300 k=355 \mathrm{MN} / \mathrm{m}$ and a dashpot $c_{0}=c_{1}=4617 \mathrm{~N} \mathrm{~s} / \mathrm{m}$.

Two configurations will be studied: a soft suspension, like in the previous examples (Fig. 26(a)), and a stiff suspension, $k^{\prime}=300 k=355 \mathrm{MN} / \mathrm{m}$, e.g. rigidity added by a stiff actuator such as a piezo-actuator (Fig. 26(b)). The damping coefficient of the suspension, modified according to the formula $c^{\prime}=0.02 \sqrt{k^{\prime} m}=6530 \mathrm{~N} \mathrm{~s} / \mathrm{m}$, is slightly larger than the damping of the structure. The dynamic equations of the system using the soft suspension read

$$
\begin{gathered}
\frac{m}{2} s^{2} x+\left(k_{1}+s c 1\right)\left(x-x_{1}\right)=0 \\
\frac{m}{2} s^{2} x_{1}+\left(k_{1}+s c_{1}\right)\left(x_{1}-x\right)+(k+s c)\left(x_{1}-x_{0}\right)=f
\end{gathered}
$$


(a)

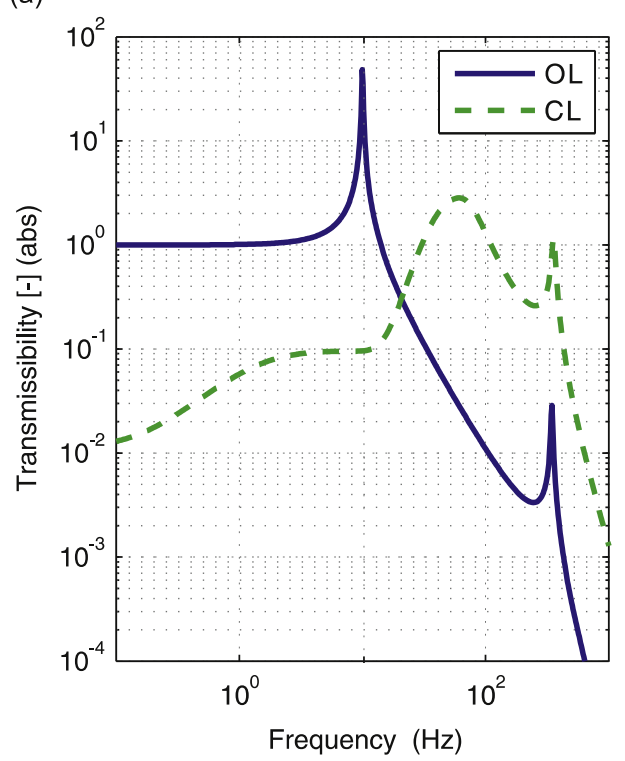

(b)

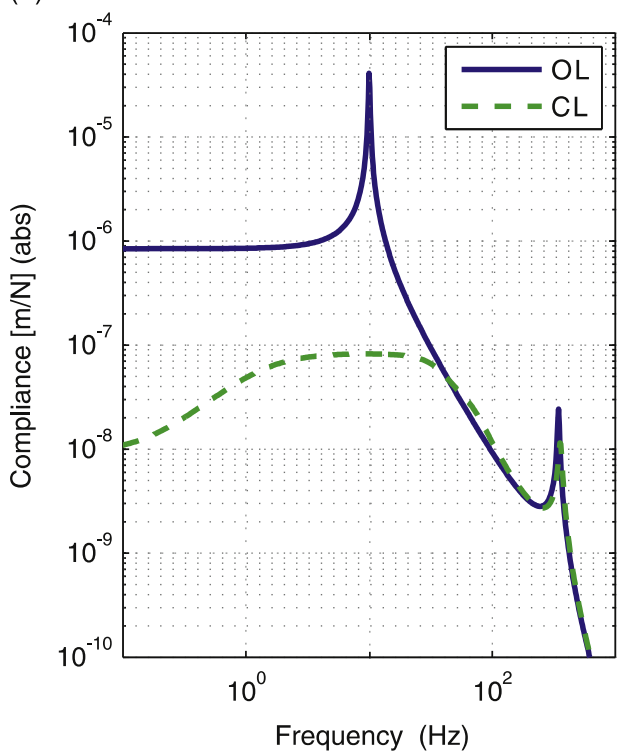

Fig. 25. Inertial sensor blended with a relative displacement sensor. (a) Transmissibility and (b) compliance of a 2 d.o.f. isolator in Open Loop (OL) and Closed Loop (CL) configuration.

(a)

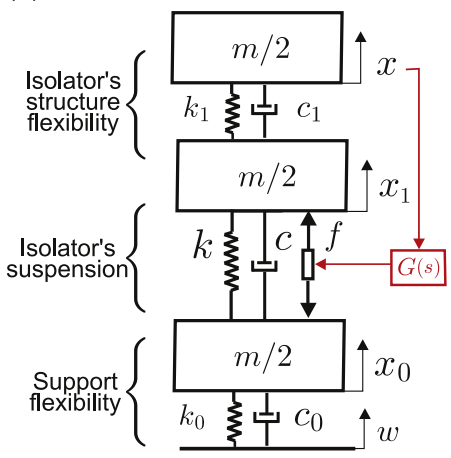

(b)

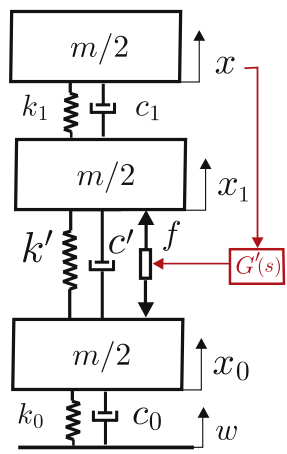

Fig. 26. Three d.o.f. model representing a flexible equipment (two upper masses), mounted on a flexible support (lower spring-mass system $k_{0}=300 k$ ): (a) soft isolator suspension; (b) stiff isolator suspension $\left(k^{\prime}=300 k\right)$.

$$
\frac{m}{2} s^{2} x_{0}+(k+s c)\left(x_{0}-x_{1}\right)+\left(k_{0}+s c_{0}\right)\left(x_{0}-w\right)=-f
$$

The equations of the system with stiff suspension are obtained upon replacing $k$ by $k^{\prime}$ and $c$ by $c^{\prime}$. For both configurations, the parameters of the flexible equipment and the flexible support remain unchanged. Throughout this section, the controller used for the soft suspension is the controller presented in Section 3, given by Eq. (25). However, as the resonance of the suspension mode has been shifted, and in order to provide a similar loop gain at low frequency, the controller used for the stiff suspension has been replaced by

$$
G^{\prime}(s)=\frac{508938009.8815(s+314.2)^{2}}{(s+1571)(s+1)}
$$

The open loop transfer function is shown in Fig. 27(a) and (b), respectively for the soft and the stiff suspension. Compared to Fig. 16, there is nearly no difference for the soft suspension, except that a new pole-zero pair appeared around $245 \mathrm{~Hz}$, related to the flexible support. The stiff isolator provides similar loop gain at low frequency, but less loop gain in the $10 \mathrm{~Hz}$ region. This could be compensated with a higher-order controller, but it is not necessary for the purpose of this discussion.

Here also, we will investigate successively the three types of blend: accelerometer, force sensor, and relative motion sensor. 
(a)
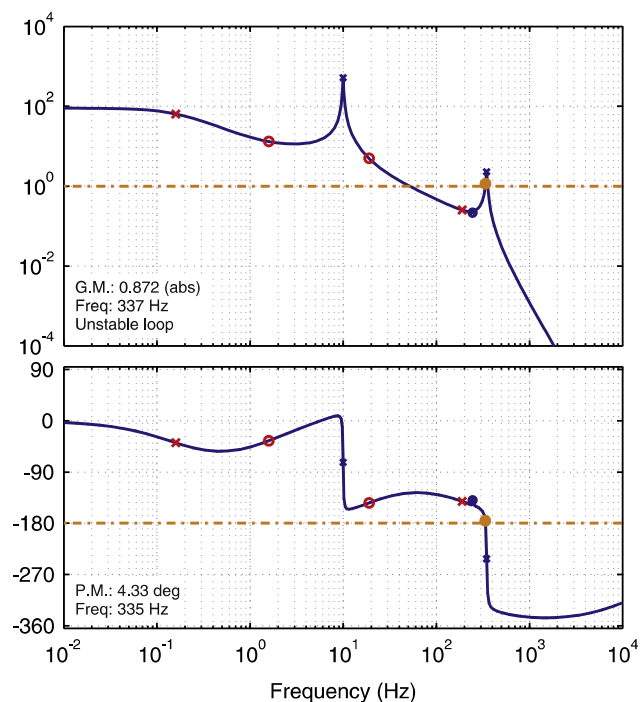

(b)

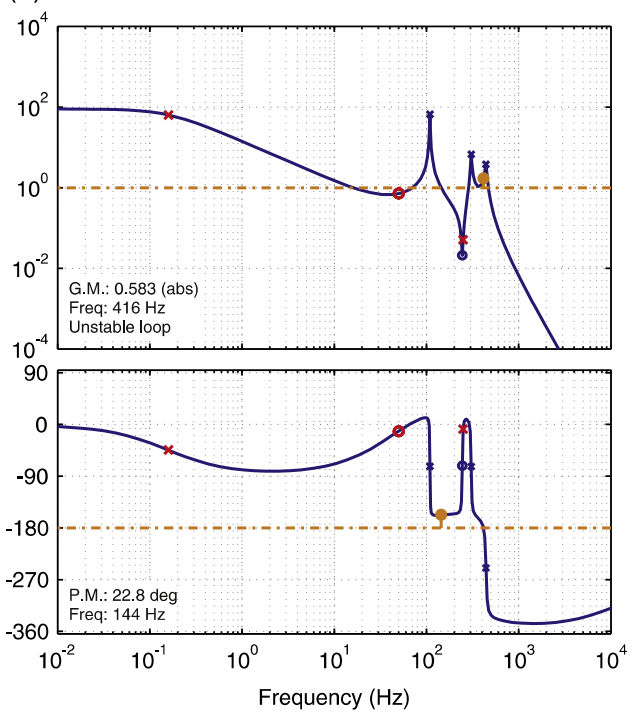

Fig. 27. Three d.o.f. isolator shown in Fig. 26. Open loop transfer function: (a) for the soft isolator suspension $G x / f$ and (b) for the stiff isolator suspension $G^{\prime} x / f$.

(a)
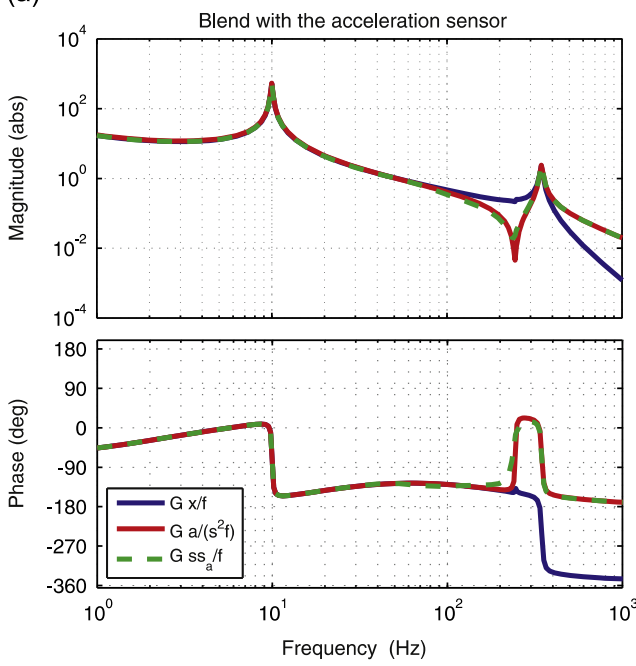

(b)
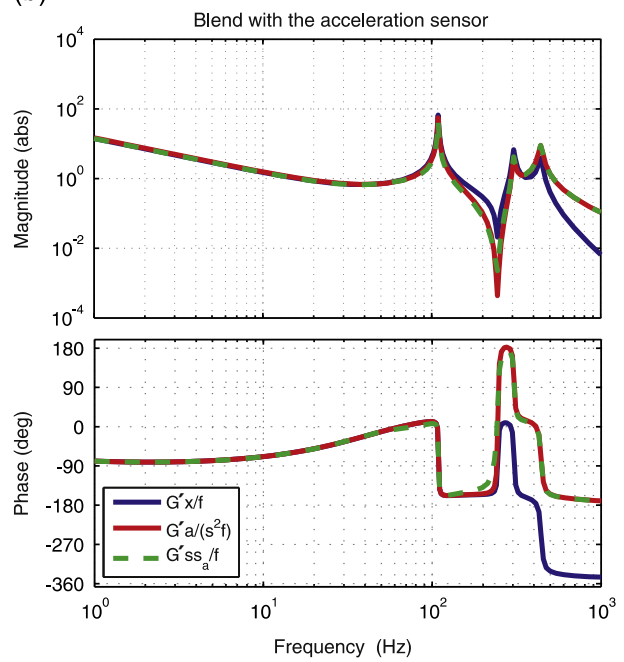

Fig. 28. Three d.o.f. isolator. Inertial sensor blended with a small accelerometer located close to the actuator. Open loop transfer functions: (a) Gx/f, $G a /\left(s^{2} f\right)$ and $G s s_{a} / f$ for the soft isolator suspension; (b) $G^{\prime} x / f, G^{\prime} a /\left(s^{2} f\right)$ and $G^{\prime} s s_{a} / f$ for the stiff isolator suspension.

\subsection{Inertial and small accelerometer}

Consider a small accelerometer mounted on the middle mass, in such a way that no structural flexibility exists between the accelerometer and the actuator. Recall that the control law for the blend is given by Eqs. (38)- (40). The open loop transfer functions between the actuator and the sensor are shown in Fig. 28.

For the soft isolator suspension (Fig. 28), the pole-zero pairs introduced by the flexibility of the support at $245 \mathrm{~Hz}$ nearly cancel each other and leave the open loop gain transfer function mostly unchanged. In this case, the system remains stable, and the closed loop performance (not shown) is similar to Fig. 19. However, for the stiff isolator suspension (Fig. 28(b)), the phase shift at $245 \mathrm{~Hz}$ is close to $+360^{\circ}$ because there are two consecutive pairs of poles. Actually, the absence of compliance between the accelerometer and the actuator is not sufficient to ensure alternating poles and zeros in the open-loop gain transfer function, because the actuator and the sensor do not form a dual pair [6]. In this example, the system is stable but the phase margin is very small.

This example illustrates that the high frequency blend with an accelerometer is an attractive method on the ground of its simplicity, but it requires a great care because it does not guaranty stability. 
(a)
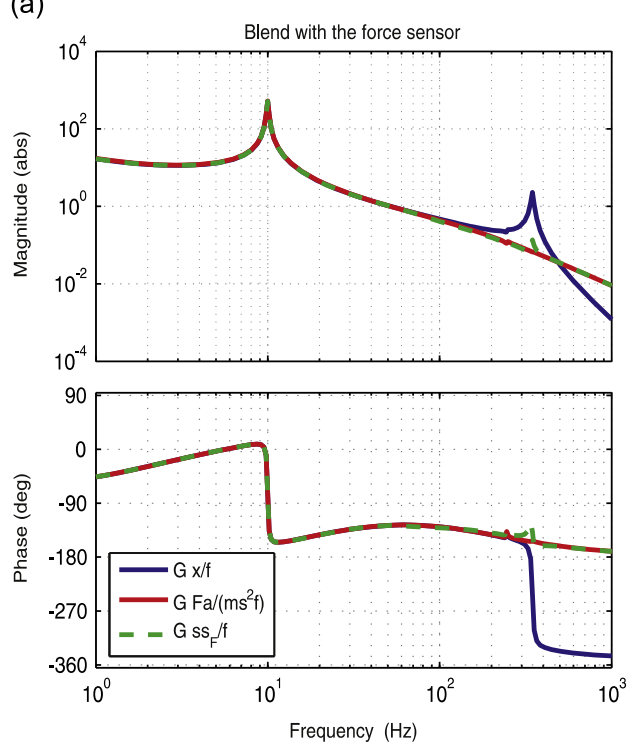

(b)
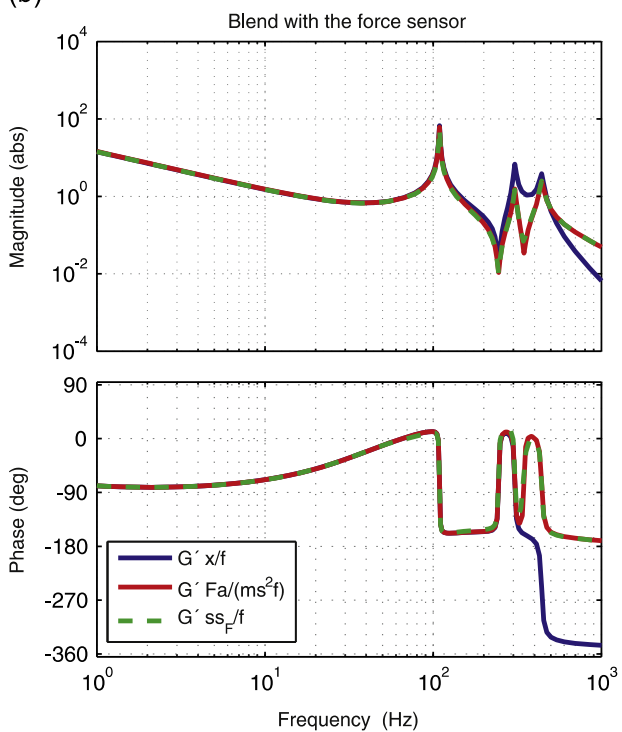

Fig. 29. Three d.o.f. isolator. Inertial sensor blended with a force sensor. Open loop transfer functions: (a) $G x / f, G F_{a} /\left(m s^{2} f\right)$ and $G s s_{F} / f$ for the soft isolator suspension; (b) $G^{\prime} x / f, G^{\prime} F_{a} /\left(m s^{2} f\right)$ and $G^{\prime} s S_{F} / f$ for the stiff isolator suspension.

(a)
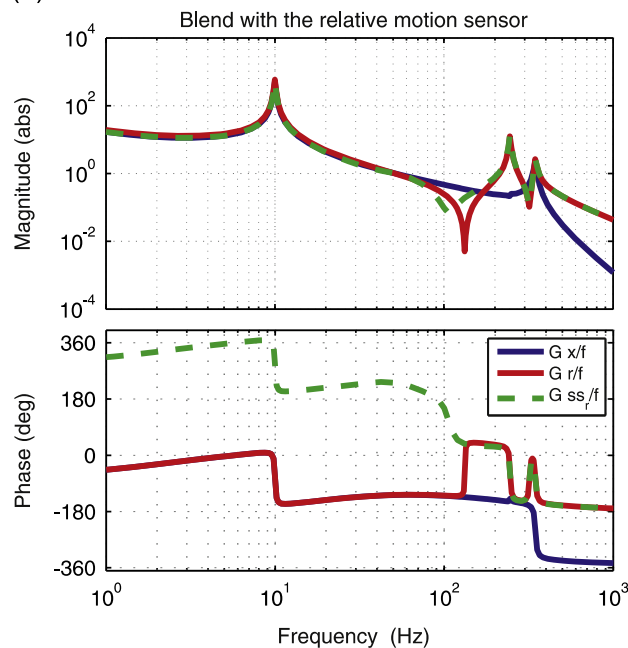

(b)
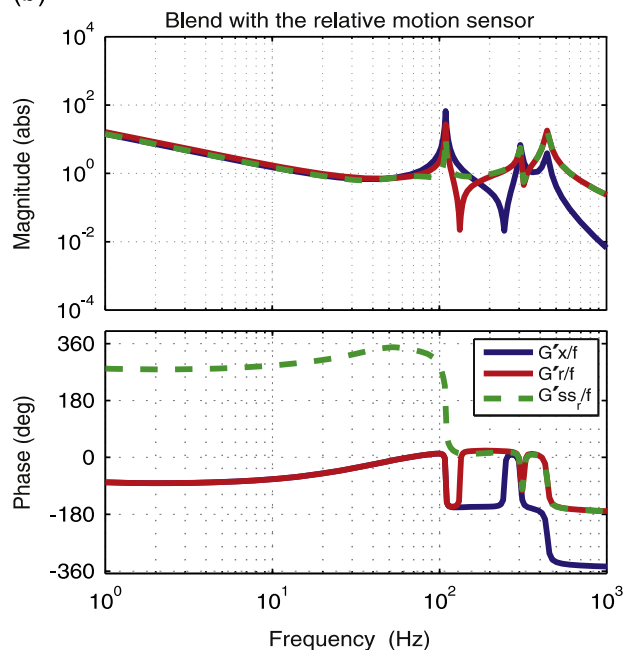

Fig. 30. Three d.o.f. isolator. Inertial sensor blended with a relative motion sensor. Open loop transfer functions: (a) $G x / f$, $G r / f$ and $G s s_{r} / f$ for the soft isolator suspension; (b) $G^{\prime} x / f, G^{\prime} r / f$ and $G^{\prime} s s_{r} / f$ for the stiff isolator suspension.

\subsection{Inertial and force sensor}

The control law is still given by Eqs. (27) and (28). Fig. 29(a) compares the open loop transfer functions $G x / f, G F_{a} /\left(m s^{2} f\right.$ ) and $G s s_{F} / f$ for the soft isolator suspension and Fig. 29(b) compares the open loop transfer functions $G^{\prime} x / f, G^{\prime} F_{a} /\left(m s^{2} f\right)$ and $G^{\prime} s s_{F} / f$ for the stiff isolator suspension. Compared to Fig. 28, one sees that the introduction of the support flexibility does not create any flip between poles and zeros when a force sensor is used. In other words, the phase remains bounded between $0^{\circ}$ and $-180^{\circ}$ for both the soft suspension $\left(G s s_{F} / f\right)$ and the stiff suspension $\left(G^{\prime} s s_{F} / f\right)$ because the actuator and the force sensor are dual.

\subsection{Inertial and relative sensor}

As for the blend with the relative sensor, the control law is still given by Eqs. (32)- (36), even though $r$ is now defined as $r=x_{1}-x_{0}$, or $r=\dot{x}_{1}-\dot{x}_{0}$, or $r=\ddot{x}_{1}-\ddot{x}_{0}$. Fig. 30(a) and (b) compares the open loop transfer functions for the soft suspension $\left(G s s_{r} / f\right)$ and the stiff suspension $\left(G^{\prime} s s_{r} / f\right)$. 
Compared to the previous cases, a sharp pair of complex zeros appeared around $130 \mathrm{~Hz}$. The frequency of the zeros is the same for both the soft isolator and the stiff isolator because it corresponds to a resonance mode where the three masses move in phase, the two bottom masses moving like if they were rigidly connected. Unlike the two d.o.f. system, the zeros interfere with the fusion filters, resulting in the phase drop visible in Fig. 30 which drives the system unstable in this case. The fusion filter could be adjusted to regain stability at the cost of further isolation degradation.

\section{Conclusion}

This paper has addressed high-frequency control-structure interaction problems in active vibration isolation systems using low-frequency, low-noise inertial sensors. The main objective of the study is to increase the seismic isolation at low frequency, i.e. increase the loop gain at low frequency, which implies that the bandwidth of the controller has to be increased as well. To this purpose, it has been proposed to fuse the inertial sensor at high frequency with sensors, collocated with the actuator. Three types of sensors have been considered: a relative motion sensor, an accelerometer and a force sensor. Their impact on the stability, the transmissibility and the compliance have been presented and compared on several models of increasing complexity.

The high-frequency fusion with a relative sensor improves the stability margins, does not modify the compliance, but compromises the transmissibility. It can be of interest for stiff suspension with little passive isolation, or for application in which the high-frequency isolation can be sacrificed to improve the stability margins. However, the flexibility of the support structure must be carefully taken into account for the design of the fusion filter. The high frequency blend with an accelerometer is an interesting mean to further increase the loop gain, because it does not affect the transmissibility and the compliance. However, as the accelerometer is not dual to the actuator, this method does not offer any guaranty of stability when the isolation stage is mounted on a flexible support. Finally, the high frequency blend with a force sensor has been also tested. It has been shown that this sensor does not suffer from the aforementioned disadvantages of the other sensors. Therefore, it can be substituted to the inertial sensor to increase the loop gain without risk for the stability and without degradation of the compliance and the transmissibility, provided that the blend is possible and that no active damping of the flexible modes is required. Although the models discussed consider a limited number of degrees of freedom to get some physical insight, the methods remain valid for a higher level of complexity, using a single actuator.

The results of this investigation will be further investigated (e.g. high frequency sensor noise, multi-degree of freedom systems, experimental validations) for application on seismic isolation systems used in Advanced LIGO gravitational wave detectors. They will also have an impact for many others applications where high performance is required, like future particle colliers, precise manufacturing machines, or satellite test facilities.

\section{Acknowledgments}

The authors gratefully acknowledge the LIGO visitors Program for making possible this collaborative work between the Université Libre de Bruxelles and the LIGO laboratory. LIGO was constructed by the California Institute of Technology and Massachusetts Institute of Technology with funding from the National Science Foundation and operates under cooperative agreement PHY-0107417. The authors also gratefully acknowledge the members of the LIGO Seismic Working Group for their comments and inspiring discussions, and particularly Jeff Kissel for carefully proof-reading the paper and making valuable comments.

\section{References}

[1] B. Abbott, Ligo: the laser interferometer gravitational-wave observatory, Reports on Progress in Physics 72 (7) (2009) 076901. URL: 〈http://stacks.iop. org/0034-4885/72/i=7/a=076901).

[2] C. Collette, S. Janssens, K. Artoos, A. Kuzmin, P. Fernandez Carmona, M. Guinchard, R. Leuxe, C. Hauviller, Nano-motion control of heavy quadrupoles for future particle colliders: an experimental validation. oai:cds.cern.ch:1423019, Nuclear Instruments and Methods in Physics Research Section A 643 (EuCARD-PUB-2011-004. 1) (2011) 95-101.

[3] C. Collette, S. Janssens, P. Fernandez-Carmona, K. Artoos, M. Guinchard, C. Hauviller, A. Preumont, Review: inertial sensors for low-frequency seismic vibration measurement, Bulletin of the Seismological Society of America 102 (4) (2012) 1289-1300.

[4] F. Matichard, LIGO vibration isolation and alignment platforms: an overview of systems, features and performance of interest for the field of precision positioning and manufacturing, ASPE Conference on Control of Precision Systems, 2013.

[5] W. Gevarter, Basic relations for control of flexible vehicles, AIAA Journal 8 (1970) 666-672.

[6] G. Martin, On the Control of Flexible Mechanical Systems, PhD Thesis, Stanford University, 1978.

[7] M. Stieber, Sensors, actuators, and hyperstability of structures, no. 10.2514/6, Guidance, Navigation and Control Conference, 1988 , pp. $1988-4057$.

[8] C. Goh, T. Caughey, On the stability problem caused by finite actuator dynamics in the collocated control of large space structures, International Journal of Control 41 (3) (1985) 787-802.

[9] F. Matichard, Prototyping, testing, and performance of the two-stage seismic isolation system for advanced ligo gravitational wave detectors, ASPE Conference on Control of Precision Systems, 2010.

[10] F. Matichard, K. Mason, R. Mittleman, B. Lantz, B. Abbott, M. McInnis, A. LeRoux, M. Hillard, C. Ramet, S. Barnum, A. Stein, S. Foley, H. Radkins, J. Kissel V. Biscans, S. Lhuillier, Dynamics enhancements of advanced ligo multi-stage active vibration isolators and related control performance improvement, Proceedings of the 24th Conference on Mechanical Vibration and Noise, Chicago, IL, USA, 2012.

[11] J. Spanos, Control-structure interaction in precision pointing servo loops, Journal of Guidance, Control and Dynamics 12 (1989) $256-263$.

[12] H. Frahm, Improved means for damping the vibrations of bodies (GB190923829A), 1909.

[13] J. Ormondroyd, J. Den Hartog, The theory of damped vibration absorber, Journal of Applied Mechanics 50 (1929) 7. 
[14] J. Yocum, L. Slafer, Control system design in the presence of severe structural dynamics interactions, Journal of Guidance, Control and Dynamics 1 (1978) $109-116$.

[15] W. Hua, Low Frequency Vibration Isolation and Alignment System for Advanced LIGO, PhD Thesis, Stanford University, 2005.

[16] G. Hauge, M. Campbell, Sensors and control of a space-based six-axis vibration isolation system, Journal of Sound and Vibration 269 (2004) 913-931.

[17] D. Tjepkema, J. Van Dijk, H.M.J.R. Soemers, Sensor fusion for active vibration isolation in precision equipment, Journal of Sound and Vibration 331 (4) (2012) 735-749.

[18] M.A. Beijen, D. Tjepkema, J. van Dijk, Two-sensor control in active vibration isolation using hard mounts, Control Engineering Practice 26 (0) (2014) $82-90$.

[19] F. Fleming, The Effect of Structure, Actuator and Sensor on the Zeroes of Collocated Structures, PhD Thesis, Massachusetts Institute of Technology, Cambridge, 1990.

[20] D. Tjepkema, Active Hard Mount Vibration Isolation for Precision Equipment, PhD Thesis, University of Twente, 2012.

[21] F. Matichard, B. Lantz, K. Mason, R. Mittleman, B. Abbott, Advanced LIGO Two-Stage Twelve-Axis Vibration Isolation and Positioning Platform. Part 2: Experimental Investigation and Tests Results, Precision engineering, arXiv:1407.6324, 2014. 